\title{
Recent Developments in Neutrino/Antineutrino-Nucleus Interactions
}

\author{
Jorge G. Morfín, ${ }^{1}$ Juan Nieves, $^{2}$ and Jan T. Sobczyk ${ }^{1}$ \\ ${ }^{1}$ Fermi National Accelerator Laboratory, Batavia, IL 60510, USA \\ ${ }^{2}$ Instituto de Fisica Corpuscular (IFIC), Centro Mixto Universidad de Valencia-CSIC, \\ Institutos de Investigacion de Paterna, E-46071 Valencia, Spain \\ Correspondence should be addressed to Jorge G. Morfín, morfin@fnal.gov
}

Received 20 July 2012; Accepted 6 September 2012

Academic Editor: Gian Luigi Fogli

Copyright (c) 2012 Jorge G. Morfín et al. This is an open access article distributed under the Creative Commons Attribution License, which permits unrestricted use, distribution, and reproduction in any medium, provided the original work is properly cited.

\begin{abstract}
Recent experimental results and developments in the theoretical treatment of neutrino-nucleus interactions in the energy range of $1-10 \mathrm{GeV}$ are discussed. Difficulties in extracting neutrinonucleon cross sections from neutrino-nucleus scattering data are explained and significance of understanding nuclear effects for neutrino oscillation experiments is stressed. Detailed discussions of the status of two-body current contribution in the kinematic region dominated by quasielastic scattering and specific features of partonic nuclear effects in weak DIS scattering are presented.
\end{abstract}

\section{Introduction}

Recent interest in neutrino interactions in the few $\mathrm{GeV}$ energy region comes from neutrino oscillation experiments and their need to reduce systematic errors. Neutrino fluxes used in contemporary long and short baseline experiments (K2 K, T2 K, MINOS, NOvA, MiniBooNE) are peaked in the $1-5 \mathrm{GeV}$ energy domain and during the last $\sim 10$ years there has been considerable theoretical and experimental activity in the investigation of neutrino cross sections in this domain with reference [1] being a good summary of the lower-energy situation. Several new cross section measurements have been performed by neutrino oscillation collaborations and there are two dedicated cross section experiments (SciBooNE and MINERvA) which have been launched at Fermilab.

Even with this degree of activity, the precision with which the basic neutrino-nucleon cross sections are known is still not better than $20-30 \%$. There are two main reasons for this: the poor knowledge of neutrino fluxes and the fact that all the recent cross section measurements have been performed on nuclear targets. It is important to recall that what 
current neutrino experiments are measuring are events that are a convolution of energydependent neutrino flux $\otimes$ energy-dependent cross section $\otimes$ energy-dependent nuclear effects. The experiments have, for example, then measured an effective neutrino-carbon cross section and to extract a neutrino-nucleon cross section from these measurements requires separation of nuclear physics effects that can be done with only limited precision. For many oscillation experiments, using the same nuclear targets for their near and far detectors is a good start. However, even with the same nuclear target in both the near and far detectors, there is no automatic cancellation of effects between detectors. This is attributable to the different effective neutrino energy distributions, due to oscillations, entering the near and far detectors. With different energy spectra, the convolution of flux $\otimes$ cross section $\otimes$ nuclear effects is different near and far and there is no automatic cancellation. For a thorough comparison of measured neutrino-nucleon cross sections with theoretical models, these convoluted effects have to be understood.

Some of the new cross section measurements raised doubts in the areas which seemed to be well understood. The list of new puzzles is quite long and seems to be expanding. What is the value of the quasielastic axial mass? How large is the two-body current contribution that can mimic genuine quasielastic interactions? How large is CC (charged current) coherent pion production at a few $\mathrm{GeV}$ neutrino energies? What is behind the large discrepancy between MiniBooNE pion production measurements and theoretical model predictions? It can be seen as a paradox that the more than 30-year old ANL and BNL low statistics deuterium pion production data, with its minimal nuclear corrections, is still used as the best source of information about the nucleon- $\Delta$ transition matrix element.

Analysis of neutrino scattering data is certainly more complicated than the analysis of electron scattering data. In the electron case, one knows exactly the initial electron energy and so also the values of energy - and momentum - transfer. It is then possible to explicitly study separate interesting kinematical regions like QE (quasielastic) peak or the $\Delta$ peak. Neutrino scattering data is always flux (often wide band!) integrated. Interacting neutrino energy must be evaluated based on kinematics of particles in the final state, taking into account detector acceptance and measurement accuracy.

For neutrino-nucleon interactions one can distinguish: charged current quasielastic (CCQE), neutral current elastic (NCEl), resonance production (RES), and more inelastic reactions up to the deep-inelastic (a rather misleading "DIS" term is often used to describe all the interactions which are neither CCQE/NCEl nor RES) domain. Quite different theoretical tools are used to model each of them. The simplest neutrino hadronic reaction is the charge current quasielastic (CCQE) interaction: $v_{\ell}+n \rightarrow \ell^{-}+p$ with two particles: charged lepton and proton in the final state. One would like to extend this definition to the neutrino-nucleus interaction occurring on bound neutrons. The obvious question arises: what is the experimental signature of CCQE on a nuclear target? The ejected proton is not necessarily seen in a detector because quite often its momentum is below the acceptance threshold. However, events with a single reconstructed charged lepton track can result from a variety of initial interactions, for example, from a two-body current interaction or from real pion production and its subsequent absorption. Similar problems arise in other type of interactions. It is becoming clear that interpretation of neutrino-nucleus interaction must rely on a careful data/Monte Carlo (MC) comparison done with reliable MC neutrino event generators. This is why we decided to include in the paper some information about development of MC event generators.

From the experimental point of view it is natural to speak about events with no pions in the final state, with only one pion, and so forth. In fact, in several recent 


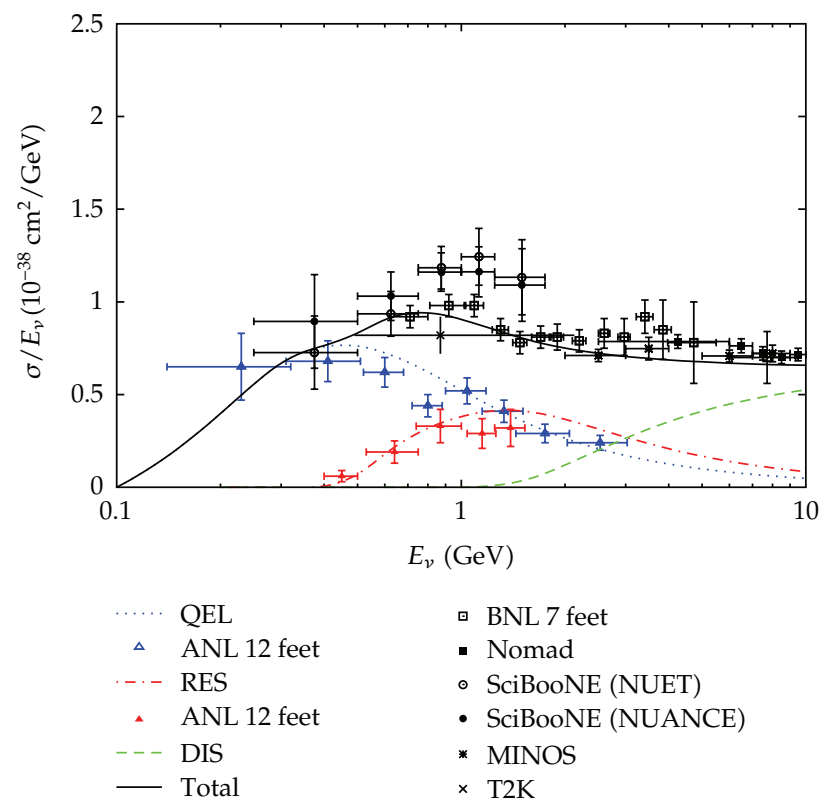

Figure 1: Breakdown of the inclusive CC muon neutrino cross section on free isoscalar target to QE, RES, and DIS contributions, as viewed by NuWro MC event generator.

experimental measurements that investigated quantities defined in this way, the dependence on assumptions of Monte Carlo event generators were minimal. To compare with the experimental data given in this format one must add contributions from various dynamical mechanisms and also to model FSI effects. Several ingredients of the theoretical models are verified simultaneously. It is clear that in order to validate a model one needs many samples of precise neutrino-nucleus scattering measurements on a variety of nuclear targets with various neutrino fluxes.

Our paper is organized as follows, we review recent inclusive measurements in the lower $E$ region and then concentrate on exclusive states in increasing $W$, the mass of the hadronic system. Due to the limited length of this paper, we do have to limit our coverage to only the most recent developments.

\section{Neutrino-Charged Current and Neutral Current Inclusive Reactions}

\subsection{Recent Measurements}

There are four recent CC inclusive neutrino and antineutrino cross section measurements in the $E_{v} \leq 10 \mathrm{GeV}$ energy region [2-5], see Figure 1. We notice a mild tension between SciBooNE and T2 K measurements. In the following sections QE, RES, and DIS contributions will be discussed separately.

\subsection{Theory: General Formulae-Outgoing Lepton Differential Cross Sections}

In this paper, we will discuss the neutrino CC or NC (neutral current) inclusive reaction:

$$
v_{\ell}(k)+A_{Z} \longrightarrow \ell\left(k^{\prime}\right)+X .
$$


The generalization of the expressions to antineutrino-induced reactions is straightforward. In the equation above, the outgoing lepton could be either a negatively charged lepton, $\ell^{-}$, of flavor $\ell$ or a neutrino $v_{\ell}$, for CC or NC processes, respectively.

The double differential cross section, with respect to the outgoing lepton kinematical variables, for the process of (2.1) is given in the Laboratory (LAB) frame by

$$
\frac{d^{2} \sigma_{v_{\ell} \ell}}{d \Omega\left(\widehat{k}^{\prime}\right) d E_{\ell}^{\prime}}=\frac{\left|\vec{k}^{\prime}\right|}{|\vec{k}|} \frac{G_{F}^{2}}{4 \pi^{2} \eta} L_{\mu \sigma}^{(v)} W^{\mu \sigma},
$$

with $\vec{k}$ and $\vec{k}^{\prime}$ the LAB lepton momenta, $E_{\ell}^{\prime}=\left(\vec{k}^{\prime 2}+m_{\ell}^{2}\right)^{1 / 2}$ and $m_{\ell}$ the energy and the mass of the outgoing lepton, $G_{F}=1.1664 \times 10^{-11} \mathrm{MeV}^{-2}$ the Fermi constant, and $L$ and $W$ the leptonic and hadronic tensors, respectively. Besides, $\eta$ takes the values 1 or 4 for CC or NC processes, respectively. The leptonic tensor is given by (in this convention, $\epsilon_{0123}=+1$ and the metric is $\left.g^{\mu \nu}=(+,-,-,-)\right)$ :

$$
L_{\mu \sigma}^{(v)}=L_{\mu \sigma}^{s}+i L_{\mu \sigma}^{a}=k_{\mu}^{\prime} k_{\sigma}+k_{\sigma}^{\prime} k_{\mu}-g_{\mu \sigma} k \cdot k^{\prime}+\mathrm{i} \epsilon_{\mu \sigma \alpha \beta} k^{\prime \alpha} k^{\beta}
$$

The hadronic tensor includes a collection of nonleptonic vertices and corresponds to the charged or neutral electroweak transitions of the target nucleon or nucleus, $i$, to all possible final states. It is thus given by

$$
W^{\mu \sigma}=\frac{1}{2 M_{i}} \bar{\sum}(2 \pi)^{3} \delta^{4}\left(P_{f}^{\prime}-P-q\right)\left\langle f\left|j_{\mathrm{CC}+, \mathrm{NC}}^{\mu}(0)\right| i\right\rangle\left\langle f\left|j_{\mathrm{CC}+, \mathrm{NC}}^{\sigma}(0)\right| i\right\rangle^{*}
$$

with $P^{\mu}$ the four momentum of the initial target, $M_{i}^{2}=P^{2}$ the target mass square, $P_{f}^{\prime}$ the total four momentum of the hadronic state $f$, and $q=k-k^{\prime}$ the four momentum transferred to the hadronic system. The bar over the sum denotes the average over initial spins.

The hadronic tensor is completely determined by six independent, Lorentz scalar and real, structure functions $W_{i}\left(q^{2}, q \cdot P\right)$,

$$
\begin{aligned}
\frac{W^{\mu \nu}}{2 M_{i}}= & -g^{\mu \nu} W_{1}+\frac{P^{\mu} P^{\nu}}{M_{i}^{2}} W_{2}+i \frac{\epsilon^{\mu \nu \gamma \delta} P_{\gamma} q_{\delta}}{2 M_{i}^{2}} W_{3}+\frac{q^{\mu} q^{\nu}}{M_{i}^{2}} W_{4} \\
& +\frac{P^{\mu} q^{\nu}+P^{v} q^{\mu}}{2 M_{i}^{2}} W_{5}+i \frac{P^{\mu} q^{\nu}-P^{v} q^{\mu}}{2 M_{i}^{2}} W_{6} .
\end{aligned}
$$

Taking $\vec{q}$ in the $z$ direction and $P^{\mu}=\left(M_{i}, \overrightarrow{0}\right)$, it is straightforward to find the six structure functions in terms of the $W^{00}, W^{x x}=W^{y y}, W^{z z}, W^{x y}$, and $W^{0 z}$ components of the hadronic tensor. After contracting with the leptonic tensor, one obtains that for massless leptons only three of them are relevant, namely,

$$
\frac{d^{2} \sigma_{v_{\ell} \ell}}{d x d y}=\frac{G_{F}^{2} M E_{v}}{\eta \pi}\left\{\left(1-y-\frac{M x y}{2 E_{v}}\right) F_{2}^{v}+x y^{2} F_{1}^{v}+y\left(1-\frac{y}{2}\right) x F_{3}^{v}\right\}
$$


with $E_{v}$ the incoming neutrino energy, $M$ the nucleon mass, $x=-q^{2} / 2 M q^{0}, y=q^{0} / E_{v}$, while the nuclear structure functions $F_{1,2,3}^{v}$ are given by, $F_{1}^{v}=2 M M_{i} W_{1}, F_{2}^{v}=2(q \cdot P) W_{2}$ and $F_{3}^{v} / M=-2(q \cdot P) W_{3} / M_{i}$. The cross section for the CC antineutrino-induced nuclear reaction is easily obtained by (i) changing the sign of the parity-violating term, proportional to $F_{3}$, in the differential cross section (this is because $L_{\mu \sigma}^{(\overline{\mathcal{v}})}=L_{\sigma \mu}^{(v)}$.), (2.6), and (ii) using $j_{\mathrm{CC}-}^{\mu}=j_{\mathrm{CC}+}^{\mu \dagger}$ in the definition/computation of the hadron tensor in (2.4)). In the case of antineutrino NC-driven processes, it is only needed to flip the sign of the term proportional to $F_{3}$ in the differential cross section, since the hadron NC is not affected.

The hadronic tensor is determined by the $W$ or $Z$ gauge boson self energy, $\Pi_{W, Z}^{\mu \rho}(q)$, in the nuclear medium. To evaluate this latter object requires a theoretical scheme, where the relevant degrees of freedom and nuclear effects could be taken into account.

In the next two sections we will discuss CCQE and pion production reactions. The general formalism described above will be used in the section devoted to DIS.

\section{Charged Current Quasielastic}

As discussed in the Introduction, we define CCQE as either the reaction on a free nucleon or on a quasi-free nucleon inside a nucleus yielding a muon and nucleon. In the case of neutrino nucleus scattering we also use the term CCQE-like reaction defined as one in which there are no pions in the final state. It then includes events with real pion production followed by absorption. Such a definition may seem awkward but as will be seen, it is close to what was experimentally measured by the MiniBooNE collaboration.

A theoretical description of the free nucleon target CCQE reaction is based on the conserved vector current (CVC) and the partially conserved axial current (PCAC) hypotheses. The only unknown quantity is the nucleon axial form-factor $G_{A}\left(Q^{2}\right)$ for which one typically assumes a dipole form $G_{A}(0)\left(1+Q^{2} / M_{A}^{2}\right)^{-2}$ with one free parameter, the axial mass $M_{A}$. The nondipole axial form factor was investigated, for example, in [6].

In the past, several measurements of $M_{A}$ were performed on a deuterium target for which most of nuclear physics complications are minimal and it seemed that the results converged to a value of the order of $1.03 \mathrm{GeV}$ [7]. There is an additional argument in favor of a similar value of $M_{A}$ coming from the weak pion-production at low $Q^{2}$. PCAC-based evaluation gives an axial mass value of $1.077 \pm 0.039 \mathrm{GeV}$ [8]. On the other hand, all of the more recent high statistics measurements of $M_{A}$, with the exception of the NOMAD higherenergy experiment, reported larger values: $\mathrm{K} 2 \mathrm{~K}$ (oxygen, $Q^{2}>0.2 \mathrm{GeV}^{2}$ ) $\rightarrow 1.2 \pm 0.12$ [9]; K2 K (carbon, $Q^{2}>0.2 \mathrm{GeV}^{2}$ ) $\rightarrow 1.14 \pm 0.11[10]$; MINOS (iron, $\left.Q^{2}>0 \mathrm{GeV}^{2}\right) \rightarrow 1.19 \pm 0.17$; MINOS (iron, $Q^{2}>0.3 \mathrm{GeV}^{2}$ ) $\rightarrow 1.26 \pm 0.17$ [11]; MiniBooNE (carbon, $Q^{2}>0 \mathrm{GeV}^{2}$ ) $\rightarrow 1.35 \pm 0.17$ [12]; MiniBooNE (carbon, $Q^{2}>0.25 \mathrm{GeV}^{2}$ ) $\rightarrow 1.27 \pm 0.14$ (for completness: NOMAD (carbon, $\left.Q^{2}>0 \mathrm{GeV}^{2}\right) \rightarrow 1.07 \pm 0.07[13]$ ).

The difference between MiniBooNE and NOMAD measurements could come from different definitions of the CCQE signal. In the case of MiniBooNE a sample of 2 subevents (Cherenkov light from muon and from decay electron) is analyzed and ejected protons are not detected. In the case of NOMAD 1-track (muon) and 2-tracks (muon and proton) samples of events are analyzed simultaneuosly. With a suitable chosen value of the formation zone parameter $\tau_{0}$ values of $M_{A}$ extracted separately from both data samples are approximately the same, see [13, Table 9]. We note that the procedures in which the formation zone concept is applied to nucleons that already exist may seem little controversial. We would like to mention also the CCQE data not yet published in peer review journals. MINOS tried to 
evaluate better the pion production background [14]. A function of $Q^{2}$ which corrects Monte Carlo (NEUGEN) RES predictions was proposed. The shape of the curve is similar to MiniBooNE's DATA/MC correction function (see below) but in the case of MiniBooNE for $Q^{2}>0.1 \mathrm{GeV}^{2}$ the correction factor is $>1$. The new MINOS best fit value of $M_{A}$ is $1.16 \mathrm{GeV}$ and the error was reduced by a factor of 3 with respect to [11]. SciBooNE showed partial results of the CCQE analysis [15]. Results are given in terms of fits for CCQE cross-section DATA/MC multiplicative factors $a_{j}$ ( $j$ label true neutrino energy bins) and a scaling factor $F_{N}$. The obtained best fit values in the neutrino energy region $E_{v} \in(0.6,1.6) \mathrm{GeV}$ are between 1.00 and 1.09 which with $F_{N}=1.02$ and the value of the axial mass used in the NEUT Monte Carlo generator $(1.2 \mathrm{GeV})$ should translate to the axial mass value $M_{A} \sim 1.25-1.3 \mathrm{GeV}$. In the SciBooNE analysis there are some instabilities in the wider region of $E_{v}$ [16, Figure 11.2]. A use of the universal background scaling factor $a_{b c g}$ for three different event samples is perhaps not sufficient (its best fit value is as large as $1.37 \mathrm{GeV}$ ).

An important antineutrino CCQE measurement was reported by MiniBooNE [17]. The DATA/MC average cross-section ratio was reported to be $1.21 \pm 0.12$ which is a surprising result because in the NUANCE carbon CCQE computations the $M_{A}$ value was set to be $1.35 \mathrm{GeV}$. In the experimental analysis, it was important to evaluate correctly neutrino contamination in the antineutrino flux. Three independent measurements indicate that the $v_{\mu}$ flux in the antineutrino beam should be scaled down by a factor of $\sim 0.8$ with an obvious important impact on the final results.

The most recent MINERvA preliminary results for CCQE antineutrino reaction are still subject to large flux normalization uncertainties but they seem to be consistent with $M_{A}=$ $0.99 \mathrm{GeV}[18]$.

\subsection{MiniBooNE Data}

In recent discussions of the CCQE, MiniBooNE measurement plays a special role. For the first time the data was presented in the form of double differential cross section in muon scattering angle and kinetic energy. Such data is the actual observable for the MiniBooNE experiment and more complete than a distribution of events in $Q^{2}$ which is calculated assuming an obviously incorrect nuclear model (the nucleon is assumed to be at rest). The signal events form a subset of events with no pions in the final state. MiniBooNE subtracted as a background, events with real pion production and subsequent absorption and also a contribution from pionless $\Delta$ decays implemented in the NUANCE MC [19] as constant fractions of $\Delta^{++}$and $\Delta^{+}$decays, following the approach of [20]. The background estimate, based on MC predictions, was later corrected by a $Q^{2}$ dependent function, which accounts for a data/MC discrepancy in the sample of events containing one $\pi^{+}$in the final state. The shape of the correction function is not well understood [21] but it has an important impact on the extracted value of $M_{A}$. The function quantifies a lack of understanding of processes

like pion absorption and can have a significant effect on the understanding of both samples of events.

MiniBooNE also provided data for the CCQE signal plus background together as the measurement of the cross section of the process in which there are no pions in the final state, the observable which is maximally independent of MC assumptions.

\subsection{Theoretical Approaches to CCQE Generalities}

Several approaches have been followed/derived to compute the relevant gauge boson absorption modes (self-energy) to describe the CCQE process. For moderate and intermediate neutrino energies, in the few $\mathrm{GeV}$ region, the most relevant ones are the absorption 


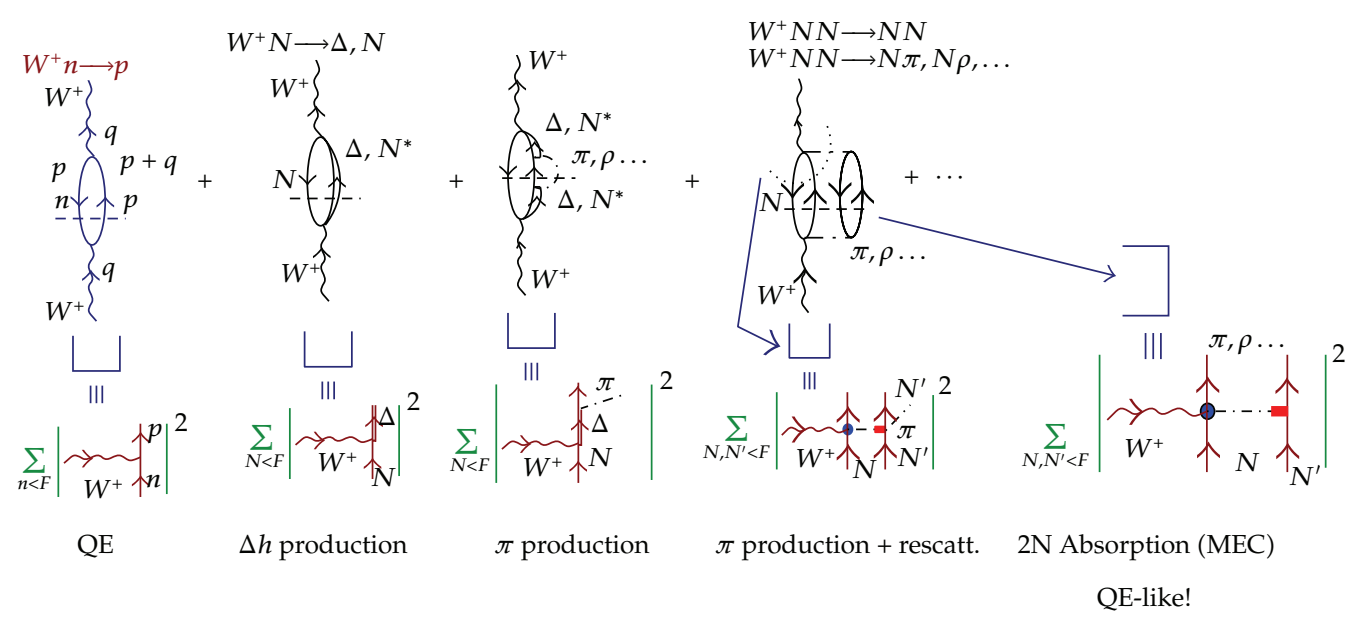

(a)

(b)

(c)

(d)

(e)

Figure 2: Diagrammatic representation of some diagrams contributing to the $W^{+}$-self energy.

by one nucleon, or a pair of nucleons or even three nucleon mechanisms, real and virtual meson $(\pi, \rho, \ldots)$ production, excitation of $\Delta$ of higher resonance degrees of freedom, and so forth (e.g., some absorption modes are depicted in Figure 2 for the case of neutrino CC processes). A review of theoretical model results can be found in [22]. Almost all approaches, used at intermediate neutrino energies, deal with hadron, instead of quarks and gluons, degrees of freedom. In addition they consider several nuclear effects such as RPA or short range correlations (SRC). The free space couplings between hadrons and/or the weak $W$ and $Z$ bosons are parametrized in terms of form factors, which are fitted to the available data on electroweak scattering off free nucleons. In the few $\mathrm{GeV}$ energy region, theoretical models rely on the impulse approximation (IA) and neutrino-nucleus CCQE interactions are viewed as a two-step process: primary interaction and final state interactions (FSI), propagation of resulting hadrons through the nucleus. The validity of the IA is usually related to typical values of the momentum transfer $q$. Experience from the electron scattering tells us that for $q>300-500 \mathrm{MeV} / \mathrm{c}$ IA-based models are able to reproduce the data well. Thus, the expectations is that for a few $\mathrm{GeV}$ neutrino interactions IA is an acceptable approach and if necessary simpler nuclear model computations can be supplemented with RPA corrections for lower momentum transfers (see below). In the neutrino nucleus cross section measurements a goal is to learn about neutrino free nucleon target scattering parameters (an obvious exception is coherent pion production). Effective parameters like sometimes discussed quasielastic axial mass $M_{A}^{\text {eff }}$ are of little use as their values can depend on the neutrino flux, target, and perhaps also on the detection technique/acceptance.

The definition of neutrino-nucleus CCQE scattering can be made more rigorous in the language of many body field theory. CCQE process originates from a first step mechanism where the gauge boson is being absorbed by just one nucleon. This corresponds to the first of the selfenergy diagrams depicted in Figure 2 (contribution (a)). This contribution, that from now on we will call genuine $\mathrm{QE}$, has been computed within different theoretical models and used to predict the corresponding outgoing lepton differential cross section.

The simplest model, commonly used in Monte Carlo event generators, is the relativistic Fermi gas (RFG) model proposed by Smith and Moniz more than 35 years ago $[23,24]$ corresponding to only one many body Feynman diagram. The model combines 
the bare nucleon physics with a model to account for Fermi motion and nucleon binding within the specific nucleus. The model can be made more realistic in many ways (when the axial mass and electromagnetic form factors are kept unchanged, the inclusion of more sophisticated nuclear effects makes the cross section generally smaller with respect to the RFG (relativistic Femi gas) model) to achieve better agreement with a broad range of electron scattering data. For example, the inclusion of a realistic joint distribution of target nucleon momenta and binding energies based on short range correlation effects leads to the spectral function (SF) approach. Spectral functions for nuclei, ranging from carbon $(A=12)$ to iron $(A=56)$ have been modeled using the local density approximation (LDA) [25], in which the experimental information obtained from nucleon knock-out measurements is combined with the results of theoretical calculations in nuclear matter at different densities, and they have been extensively validated with electron scattering data. Calculations by Benhar and Meloni [26] and Ankowski and Sobczyk [27] show that the SF effects moderately modify the muon neutrino differential cross sections, and they lead to reductions of the order of $15 \%$ in the total cross sections. This is corroborated by the results obtained within the semiphenomenological model (density dependent mean-field potential in which the nucleons are bound) [28] employed within the GiBUU model to account for these effects.

Inclusion of nucleon-nucleon long-range correlations leads to RPA (random phase approximation) which improves predictions at lower momentum transfers (and also low $Q^{2}$ ). RPA corrections have been discussed by many authors in the past and recently included in computations of three groups (IFIC, Lyon, and Aligarh (the Aligarh group uses a similar approach to that derived in $[29,30]$, but with some simplifications that though well suited to study the related process of muon capture in nuclei, might not be totally appropriate for the case of larger energies and momenta being transferred to the nucleus (see the discussion in $[29,30]))$ in $[29-34]$, respectively. When the electroweak interactions take place in nuclei, the strengths of electroweak couplings may change from their free nucleon values due to the presence of strongly interacting nucleons. Indeed, since the nuclear experiments on $\beta$ decay in the early 1970s [35, 36], the quenching of axial current is a well-established phenomenon. The RPA resummation accounts for the medium polarization effects in the 1 p1h contribution (Figure 2(a)) to the $W$ and $Z$ selfenergy by substituting it by a collective response as shown diagrammatically in Figure 3(a). Evaluating these effects, requires an inmedium baryon-baryon effective force, which in both sets (IFIC and Lyon) of calculations was successfully used/tested in previous works on inclusive nuclear electron scattering. RPA effects are important as can be appreciated in Figure 3(b). In this plot, we show results from both IFIC and Lyon models, presented in $[37,38]$, respectively, for the CC quasielastic $v_{\mu}-{ }^{12} \mathrm{C}$ double differential cross sections convoluted with the MiniBooNE flux [39]. There, we also see that predictions of both groups for these genuine QE contribution, with and without RPA effects, turn out to be in a quite good agreement. Finally, it is important to stress also that RPA corrections strongly decrease as the neutrino energy increases, while its effects should account for a low $Q^{2}$ deficit of CCQE events reported by several experimental groups (see Figures 3(c) and 3(d)). Continuum RPA (CRPA) computations for neutrino scattering were performed by the Ghent group [40].

\subsubsection{Other Theoretical Developments}

In [41-43] the bound-state wave functions are described as self-consistent Dirac-Hartree solutions, derived within a relativistic mean field approach by using a Lagrangian containing $\sigma$ and $\omega$ mesons [44]. This scheme also accounts for some SF effects. Moreover, these models 


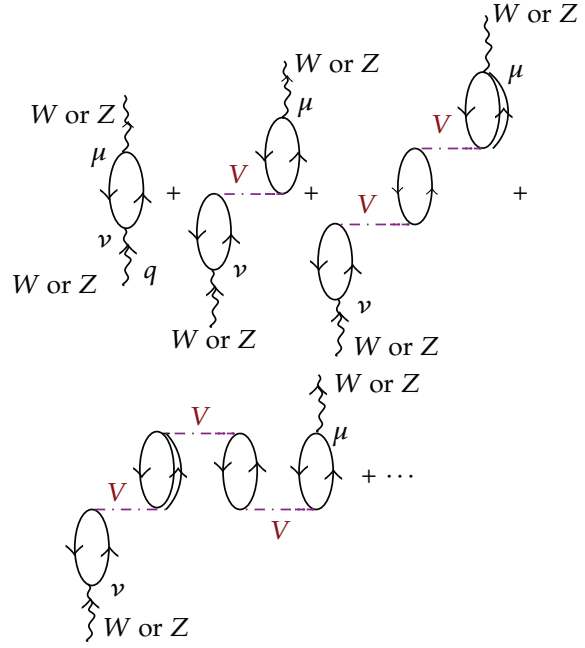

(a)

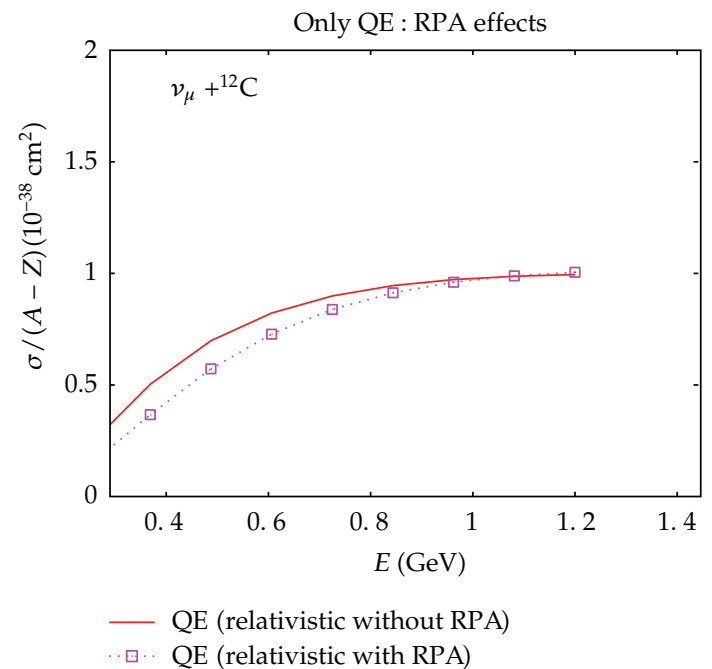

(c)

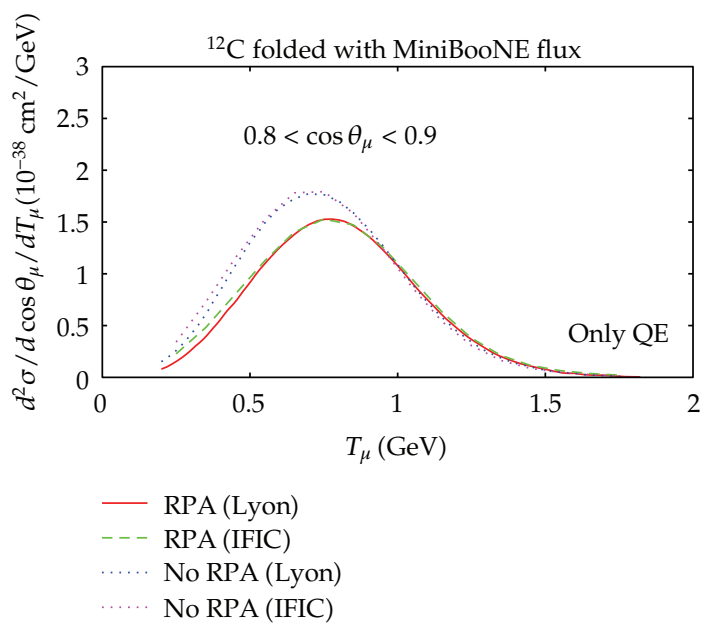

(b)

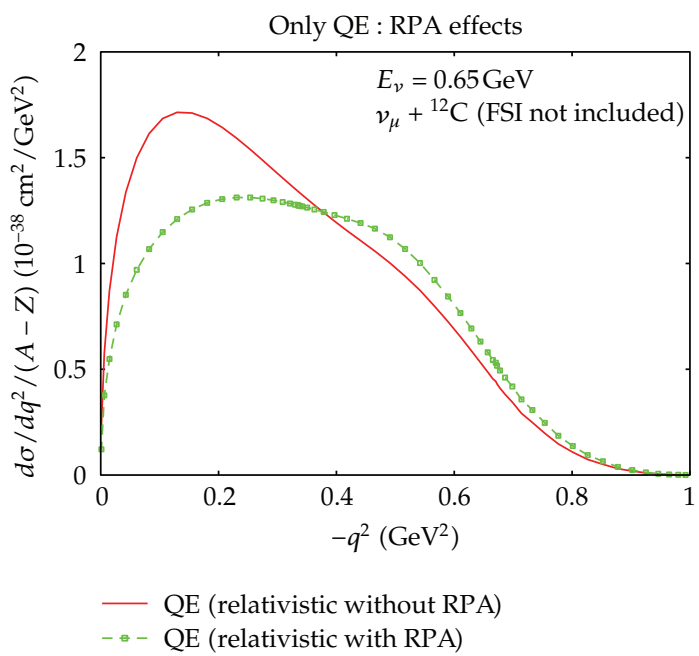

(d)

Figure 3: (a) Set of irreducible diagrams responsible for the polarization (RPA) effects in the $1 \mathrm{p} 1 \mathrm{~h}$ contribution to the $W$ or $Z$ self energies. (b) MiniBooNE flux-averaged CC quasielastic $v_{\mu}{ }^{12} \mathrm{C}$ double differential cross section per neutron for $0.8<\cos \theta_{\mu}<0.9$ as a function of the muon kinetic energy. (c, d) Different theoretical predictions for muon neutrino CCQE total cross section off ${ }^{12} \mathrm{C}$, as a function of the neutrino energy (c) and $q^{2}(\mathrm{~d})$, obtained from the relativistic model of $[29,30]$. In all cases $M_{A} \sim 1.05 \mathrm{GeV}$.

also incorporate the FSI between the ejected nucleon and the residual nucleus. The final nucleon is described either, as a scattering solution of the Dirac equation [42, 43] in the presence of the same relativistic nuclear mean field potential applied to the initial nucleon, or adopting a relativistic multiple-scattering Glauber approach [41].

The relativistic Green's function model [45] would be also appropriate to account for FSI effects between the ejected nucleon and the residual nucleus for the inclusive scattering, where only the outgoing lepton is detected. There, final-state channels are included, and the flux lost in each channel is recovered in the other channels just by the imaginary part of an empirical optical potential and the total flux is thus conserved. 
Another interesting approach starts with a phenomenological model for the neutrino interactions with nuclei that is based on the superscaling behavior of electron scattering data. Analysis of inclusive $\left(e, e^{\prime}\right)$ data have demonstrated that for momentum transfers $q>\sim 500 \mathrm{MeV} / \mathrm{c}$ at energy transfers below the QE peak superscaling is fulfilled rather well $[46,47]$. The general procedure consists on dividing the experimental $\left(e, e^{\prime}\right)$ cross section by an appropriate single-nucleon cross section to obtain the experimental scaling function, which is then plotted as a function of a certain scaling variable for several kinematics and for several nuclei. If the results do not depend on the momentum transfer $q$, then scaling of the first kind occurs, if there is no dependence on the nuclear species, one has scaling of the second kind. The simultaneous occurrence of scaling of both kinds is called superscaling. The superscaling property is exact in the RFG models, and it has been tested in more realistic models of the $\left(e, e^{\prime}\right)$ reaction. The superscaling approach (SuSA) is based on the assumed universality of the scaling function for electromagnetic and weak interactions [48]. The scaling function thus determined from $\left(e, e^{\prime}\right)$ data is then directly taken over to neutrino interactions $[48,49]$. There are no RPA correlations or SF corrections explicitly taken into account, but they may be contained in the scaling function. Nevertheless, such approach is far from being microscopic. Moreover, it is difficult to estimate its theoretical uncertainties, as for example to what extent the quenching of the axial current, that is due to RPA corrections, is accounted for by means of scaling functions determined in $\left(e, e^{\prime}\right)$ experiments, which are driven by the vector current.

\subsubsection{Theoretical Models versus MiniBooNE 2D Data}

The MiniBooNE data [12] have been quite surprising. Firstly, the absolute values of the cross section are too large as compared to the consensus of theoretical models [22, 50]. Actually, the cross section per nucleon on ${ }^{12} \mathrm{C}$ is clearly larger than for free nucleons. Secondly, their fit to the shape (excluding normalization) of the $Q^{2}$ distribution done within the RFG model leads to the axial mass, $M_{A}=1.35 \pm 0.17 \mathrm{GeV}$, much larger than the previous world average $(\approx 1.03 \mathrm{GeV})[8,13]$. Similar results have been later obtained analyzing MiniBooNE data with more sophisticated treatments of the nuclear effects that work well in the study of electron scattering. For instance, $[51,52]$ using the impulse approximation with state of the art spectral functions for the nucleons fail to reproduce data with standard values of $M_{A}$. Large axial mass values have also been obtained in [53] where the 2D differential cross section was analyzed for the first time using RFG model and spectral function. Similar results were obtained in [54], where the data have been analyzed in a relativistic distorted-wave impulse approximation supplemented with a RFG model.

\subsection{Multinucleon Mechanisms}

A plausible solution to the large axial mass puzzle was firstly pointed out by Martini et al. [32, 33] (the papers of Martini et al. are based on the older investigation by Marteau [55]. The relevant features of the model were known already at the end of 1990s and at that time the goal was to understand better SuperKamiokande atmospheric neutrino oscillation signal), and later corroborated by the IFIC group $[37,56]$. In the MiniBooNE measurement of [12], QE is related to processes in which only a muon is detected in the final state. As was already discussed above, besides genuine QE events, this definition includes multinucleon processes (Figure 2(e) (note that the intermediate pion in this term is virtual and it is part 


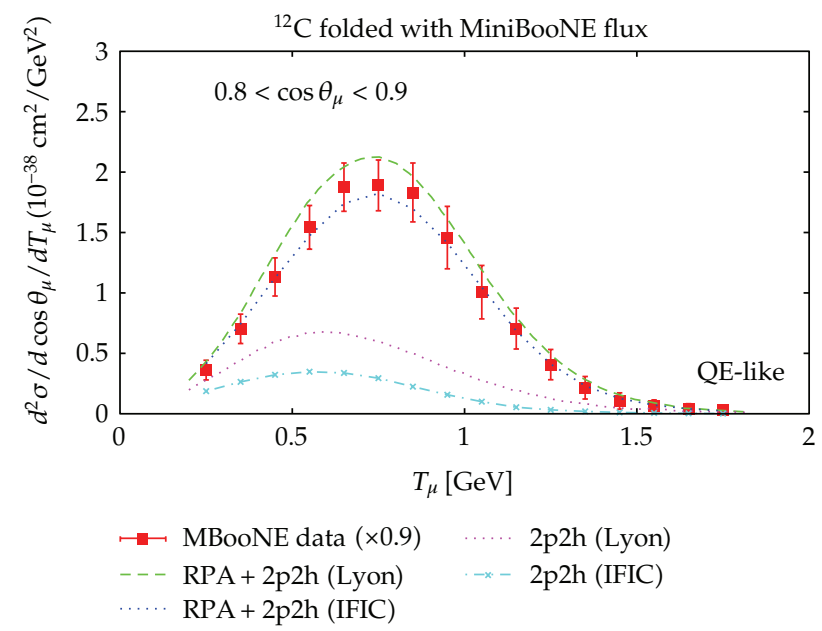

Figure 4: MiniBooNE flux-averaged CC quasielastic $v_{\mu}-{ }^{12} \mathrm{C}$ double differential cross section per neutron for $0.8<\cos \theta_{\mu}<0.9$, as a function of the muon kinetic energy. Experimental data from [12] are multiplied by 0.9 . In all the cases $\mathrm{M}_{\mathrm{A}} \sim 1.05 \mathrm{GeV}$.

of the $\Delta N \rightarrow N N$ interaction inside of the nucleus. Indeed, one should consider a full interaction model for the in medium baryon-baryon interaction), where the gauge boson is being absorbed by two or more nucleons, and others like real pion production followed by absorption (Figures 2(c) and 2(d)). The MiniBooNE analysis of the data attempts to correct (through a Monte Carlo estimate) for some of these latter effects, such as real pion production that escapes detection through reabsorption in the nucleus leading to multinucleon emission. But, it seems clear that to describe the data of [12], it is necessary to consider, at least, the sum of the selfenergy diagrams depicted in Figures 2(a) and 2(e). Those correspond to the genuine QE (absorption by just one nucleon), and the multinucleon contributions, respectively. These two mechanisims contribute to the CCQE-like cross section (also for simplicity, we will often refer to the multinucleon mechanism contributions, though they include effects beyond gauge boson absorption by a nucleon pair, as $2 \mathrm{p} 2 \mathrm{~h}$ (two-particle hole) effects).

The inclusion of the $2 \mathrm{p} 2 \mathrm{~h}$ contributions enables $[37,38]$ the double differential cross section $d^{2} \sigma / d E_{\mu} d \cos \theta_{\mu}$ and the integrated flux unfolded cross section (we should warn the reader here, because of the multinucleon mechanism effects, the algorithm used to reconstruct the neutrino energy is not adequate when dealing with quasielastic-like events, a distortion of the total flux unfolded cross section shape could be produced. We will address this point in Section 3.5) measured by MiniBooNE, to be described with values of $M_{A}$ (nucleon axial mass) around $1.03 \pm 0.02 \mathrm{GeV}[8,13]$. This is reassuring from the theoretical point of view and more satisfactory than the situation envisaged by some other works that described the MiniBooNE data in terms of a larger value of $M_{A}$ of around 1.3-1.4 GeV, as mentioned above.

\subsubsection{Similarites and Differences between Multinucleon Ejection Models}

As shown in Figures 3(a) and 3(b), the IFIC group predictions [37, 56] for QE cross sections agree quite well with those obtained in [32, 33, 38] (Lyon group). However, both above presented approaches considerably differ (about a factor of two) in their estimation of the size of the multinucleon effects, as can be appreciated in Figure 4. IFIC predictions, when 
the $2 \mathrm{p} 2 \mathrm{~h}$ contribution is included, favor a global normalization scale of about 0.9 (see [37]). This is consistent with the MiniBooNE estimate of a total normalization error as $10.7 \%$. The IFIC evaluation in $[37,56]$, of multinucleon emission contributions to the cross section is fully microscopical and it contains terms, which were either not considered or only approximately taken into account in $[32,33,38]$. Indeed, the results of these latter works rely on some computation of the $2 \mathrm{p} 2 \mathrm{~h}$ mechanisms for the $\left(e, e^{\prime}\right)$ inclusive reaction [57], which results are simply used for neutrino-induced processes without modification. Thus, it is clear that these latter calculations do not contain any information on axial or axial-vector contributions (the evaluation of the nuclear response induced by these $2 \mathrm{p} 2 \mathrm{~h}$ mechanisms carried out in [32] is approximated, as acknowledge there. Only, the contributions in [32] that can be cast as a $\Delta$-selfenergy diagram should be quite similar to those derived in [56] by the IFIC group, since in both cases the results of [20] for the $\Delta$-selfenergy are used). For antineutrinos the IFIC model predicts, contrary to the results of the Lyon group, also a sizeable effect of $2 \mathrm{p} 2 \mathrm{~h}$ excitations.

Another microscopic approach to $2 \mathrm{p} 2 \mathrm{~h}$ excitations was proposed by Amaro et al. These authors have used the empirical $\left(e, e^{\prime}\right)$ SuSA scaling function to describe the CCQE MinibooNE data, including some $2 \mathrm{p} 2 \mathrm{~h}$ contributions due to MEC (meson exchange currents) $[58,59]$. The approach, used in these latter works, to evaluate the $2 \mathrm{p} 2 \mathrm{~h}$ effects, though fully relativistic, does not contain the axial contributions. The authors of [58, 59] also find an increase of the inclusive cross section for neutrinos; at forward muon angles the calculations come close to the data, but the MEC contributions die out fast with increasing angle so that the cross section is significantly underestimated at backward angles. As a consequence, the energy-separated (flux unfolded) cross section obtained for the MiniBooNE experiment, while being higher than that obtained from SuSA alone still, underestimates the experimental result even when $2 \mathrm{p} 2 \mathrm{~h}$ contributions are added. Recently, a strong difference between neutrino and antineutrino cross sections has been obtained within this model, with the $2 \mathrm{p} 2 \mathrm{~h}$ effects being significantly larger for antineutrinos than for neutrinos [59].

Two other effective models to account for MEC $/ 2 \mathrm{p} 2 \mathrm{~h}$ effects have been proposed by Bodek et al. [60] (transverse enhancement model (TEM)) and Lalakulich et al. [61]. The TEM can easily be implemented in MC event generators [62]. It assumes that it is sufficient to describe properly an enhancement of the transverse electron QE response function keeping all other ingredients as in the free nucleon target case. Thus, some effective proton and neutron magnetic form factors are fitted to electron-nucleus data and later they are used, together with the free nucleon axial current, to study CCQE processes. It is to say, the TEM assumes that there are no nuclear medium effects (RPA, 2p2h mechanisms, etc...) affecting those nuclear response functions induced by the nucleon axial-vector current. Despite of a certain phenomenological success to describe the MiniBooNE data $[60,62]$, such assumption seems quite unjustified.

In the model of [61], the multinucleon mechanism contributions are parametrized as phase space multiplied by a constant which is fitted to the difference of the energy-separated MiniBooNE data and the calculated QE cross section. RPA effects are not taken into account in [61]. Since these tend to lower the cross section in particular at forward muon angles, the model of [61] underestimates the contributions of 2p2h effects there. Indeed, the authors of this reference find that the shape and over-all size of the $2 \mathrm{p} 2 \mathrm{~h}$ contribution turns out to be rather independent of the muon angle. This is in sharp contrast with the microscopical results obtained within the IFIC $[37,56]$ and SuSa models [59], that find the 2p2h contribution becomes significantly less important as the muon scattering angle increases. 


\subsubsection{Perspectives to Measure the MEC/2p2h Contribution}

The unambiguous experimental measurement of the MEC contribution to the CC inclusive cross section can be made by detecting hadrons in the final state. All the microscopic models provide up to now only the MEC/2p2h contribution to the muon inclusive $2 \mathrm{D}$ differential cross section: $d^{2} \sigma_{v_{\ell} \ell} / d \Omega\left(\widehat{k}^{\prime}\right) d E_{\ell}^{\prime}$. Such models cannot describe detailed exclusive cross sections (looking into the nucleon side), as explicit FSI effects, that modify the outgoing nucleon spectra, have not been addressed yet in these microscopical models. It is reasonable to assume that at the level of the primary reaction mechanism, they produce only slight changes in $d^{2} \sigma_{v_{\ell} \ell} / d \Omega\left(\widehat{k}^{\prime}\right) d E_{\ell^{\prime}}^{\prime}$ leaving almost unchanged the integrated cross sections $[26,27]$.

A model to describe hadrons in the final state was proposed in [62]. It was implemented in the NuWro MC event generators and its predictions were used in the analysis of recent MINERvA antineutrino CCQE data.

In the papers $[61,62]$ various observables are discussed which can be used to detect MEC contribution. One option is to look at proton pairs in the final state. Another possibility is to investigate the distribution of visible energy which allows to include contributions from protons below reconstruction threshold. The basic intuition from the electron scattering is that MEC events populate the region between $\mathrm{QE}$ and $\Delta$ peaks. Typically, to have an MEC event more energy must be transferred to the hadronic system than for a CCQE one. However, it should be stressed that the precision with which FSI effects are currently handled in MC codes can make such a measurement difficult. During the last few years FSI studies were focused on pions only [63] aiming at understanding recent pion production data on nuclear targets [64]. Nucleons in the final state were never studied with a similar precision so there is less data to benchmark nucleon FSI effects.

\subsection{Monte Carlo Event Generators}

Monte Carlo codes (GENIE, NuWro, Neut, Nuance, etc.) describe CCQE events using a simple RFG model, with FSI effects implemented by means of a semiclassical intranuclear cascade. NuWro offers also a possibility to run simulations with spectral function and an effective momentum-dependent nuclear potential. It is also by now the only MC generator with implementation of MEC dynamics. Since the primary interaction and the final state effects are effectively decoupled, FSI do not change the total and outgoing lepton differential cross sections.

\subsection{Neutrino Energy Reconstruction}

Neutrino oscillation probabilities depend on the neutrino energy, unknown for broad fluxes and often estimated from the measured angle and energy of the outgoing charged lepton $\ell$ only. This is the situation of the experiments with Cherenkov detectors where protons in the final state are usually below the Cherenkov threshold. Then, it is common to define a reconstructed neutrino energy $E_{\text {rec }}$ (neglecting binding energy and the difference of proton and neutron masses) as follows:

$$
E_{\mathrm{rec}}=\frac{M E_{\ell}-m_{\ell}^{2} / 2}{M-E_{\ell}+\left|\vec{p}_{\ell}\right| \cos \theta_{\ell}}
$$


which would correspond to the energy of a neutrino that emits a lepton, of energy $E_{\ell}$ and three-momentum $\vec{p}_{\ell}$, with a gauge boson $W$ being absorbed by a free nucleon of mass $M$ at rest in a CCQE event. Each event contributing to the flux averaged double-differential cross section $d \sigma / d E_{\ell} d \cos \theta_{\ell}$ defines unambiguously a value of $E_{\text {rec. }}$. The actual ("true") energy, $E$, of the neutrino that has produced the event will not be exactly $E_{\text {rec }}$. Actually, for each $E_{\text {rec, }}$ there exists a distribution of true neutrino energies that give rise to events whose muon kinematics would lead to the given value of $E_{\text {rec }}$. In the case of genuine QE events, this distribution is sufficiently peaked (the Fermi motion broadens the peak and binding energy shifts it a little) around the true neutrino energy to make the algorithm in (3.1) accurate enough to study the neutrino oscillation phenomenon [65] or to extract neutrino flux unfolded CCQE cross sections from data (assuming that the neutrino flux spectrum is known) [66, 67]. The effect of this assumption on the much more demanding measurement of CP-violation effects is currently being evaluated.

However, and due to presence of multinucleon events, there is a long tail in the distribution of true energies associated to each $E_{\text {rec }}$ that makes the use of (3.1) unreliable. The effects of the inclusion of multinucleon processes on the energy reconstruction have been noticed in [62] and investigated in [66], within the Lyon 2p2h model and also estimated in [68], using the simplified model of [61] for the multinucleon mechanisms. This issue has been more recently also addressed in the context of the IFIC 2p2h model in [67], finding results in a qualitative agreement with those of $[66,68]$.

In [67] it is also studied in detail the ${ }^{12} \mathrm{C}$ unfolded cross section published in [12]. It is shown there that the unfolding procedure is model dependent. Moreover, it is also shown that the MiniBooNE published CCQE cross section as a function of neutrino energy differs from the real one. This is because the MiniBooNE analysis assumes that all the events are QE. The authors of [67] finally conclude that the MiniBooNE unfolded cross section exhibits an excess (deficit) of low (high) energy neutrinos, which is mostly an artifact of the unfolding process that ignores multinucleon mechanisms.

\subsection{NC Elastic}

MiniBooNE has also measured flux integrated NC elastic reaction cross section [69]. Using these data, the best fit value of the axial mass was found te be $M_{A}=1.39 \pm 0.11 \mathrm{GeV}$. The measurement was possible because the MiniBooNE Cherenkov detector can observe also scintillation light from low momentum nucleons. An attempt was done to measure the nucleon strange quark component using the proton enriched sample of events with a result consistent with zero: $\Delta s=0.08 \pm 0.26$.

\subsubsection{Theoretical Considerations}

The MiniBooNE NCEl data were analyzed in [70]. The fit was done to the $Q^{2}$ distribution of events with the best fit value of $M_{A}$ equal to $1.28 \pm 0.05 \mathrm{GeV}$. Moreover the authors of [71] concluded that axial mass as large as $1.6 \mathrm{GeV}$ is still too small to reproduce the MiniBooNE $\mathrm{NCEl}$ data. Critical discussion of this statement can be found in [72].

\section{The Resonance Region}

In the RES region the degrees of freedom are hadronic resonances, with the most important being the $\Delta(1232)$. Typical final states are those with a single pion. During the last five 
years several new pion production measurements have been performed. In all of them the targets were nuclei (most often carbon) and interpretation of the data in terms of the neutrino-nucleon cross section needed to account for nuclear effects, impossible to do in a model independent manner. Because of that it has become a standard that the published data include nuclear effects with most uncertain FSI. Perhaps not surprisingly, in several papers old deuterium ANL and BNL pion production data were reanalyzed aiming to better understand the pion production reaction on free nucleons. Theoretical models became more sophisticated and the major improvement was a development of well justified mechanisms for the nonresonant contribution in the $\Delta$ region. Some papers addressed the problem of higher resonances, a topic which will be investigated experimentally with future MINERvA results. On the other hand, there has been a lot of activity in the area of the coherent pion production and this subject will be discussed separately.

\subsection{Experimental Results}

\subsection{1. $N C \pi^{0}$}

Neutral current $\pi^{0}$ production $\left(\mathrm{NC} \pi^{0}\right)$ is a background to $v_{e}$ appearance oscillation signal. One is interested in a $\pi^{0}$ leaving the nucleus and recent experimental data are given in this format with all the FSI effects included. Signal events originate mostly from: NC1 $\pi^{0}$ primary interaction with a $\pi^{0}$ not being affected by FSI and NC1 $\pi^{+}$primary interaction with the $\pi^{+}$being transformed into $\pi^{0}$ in a charge exchange FSI reaction. An additional difficulty in interpreting the $\mathrm{NC} \pi^{0}$ production comes from a coherent $(\mathrm{COH})$ contribution. In the case of MiniBooNE flux neutrino-carbon reactions $\left(\left\langle E_{v}\right\rangle \sim 1 \mathrm{GeV}\right)$ it is estimated to account for $\sim 20 \%$ of signal events [73].

Four recent measurements of $\mathrm{NC} \pi^{0}$ production (K2K [74], MiniBooNE neutrinos, MiniBooNE antineutrinos [75], SciBooNE [76]) are complementary. They use three different fluxes: (K2K, Fermilab Booster neutrinos, and antineutrinos) and three targets: $\mathrm{H}_{2} \mathrm{O}(\mathrm{K} 2 \mathrm{~K})$, $\mathrm{CH}_{2}$ (MiniBooNE), and $\mathrm{C}_{8} \mathrm{H}_{8}$ (SciBooNE). MiniBooNE presented the results in the form of absolutely normalized cross section while $\mathrm{K} 2 \mathrm{~K}$ and SciBooNE reported only the ratios $\sigma\left(\mathrm{NC} 1 \pi^{0}\right) / \sigma(\mathrm{CC})$. There is an important difference in what was actually measured: $\mathrm{K} 2 \mathrm{~K}$ and MiniBooNE present their results as measurements of final states with only one $\pi^{0}$ and no other mesons. SciBooNE defines the signal as states with at least one $\pi^{0}$ in the final state so that a contamination from $1 \pi^{0} 1 \pi^{ \pm}, 2 \pi^{0}$, and $>2 \pi$ (with $>1 \pi^{0}$ ) final states is included and its fraction can be estimated to be $17 \%$ [64]. Final results are presented as flux averaged distributions of events as a function of the $\pi^{0}$ momentum, and in the case of MiniBooNE and SciBooNE also as a function of the $\pi^{0}$ production angle.

\subsection{2. $\mathrm{CC} \pi^{+}$}

MiniBooNE measured $\mathrm{CC} 1 \pi^{+}$production cross sections, where the signal is defined as exactly one $\pi^{+}$in the final state with no other mesons [77]. A variety of flux integrated differential cross sections, often double differential were reported in $Q^{2}$ and final state particles momenta. Also absolute $\pi^{+}$production cross sections as a function of neutrino energy are provided in [77]. The cross section results are much larger than NUANCE MC predictions and the difference is on average 23\%. In Figure 5(a) GiBUU and NuWro predictions for $\mathrm{CC} \pi^{+}$are compared to the MiniBooNE data. 


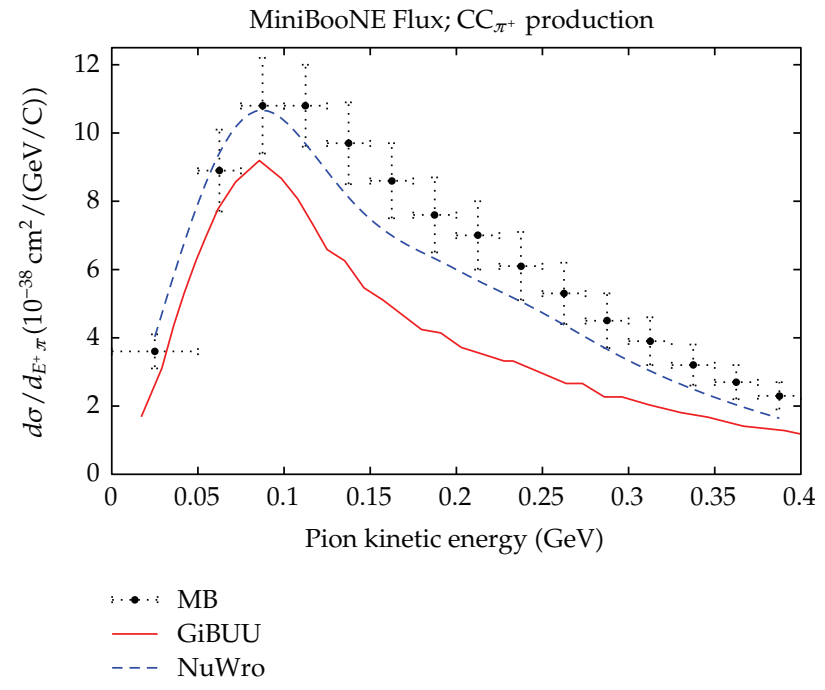

(a)

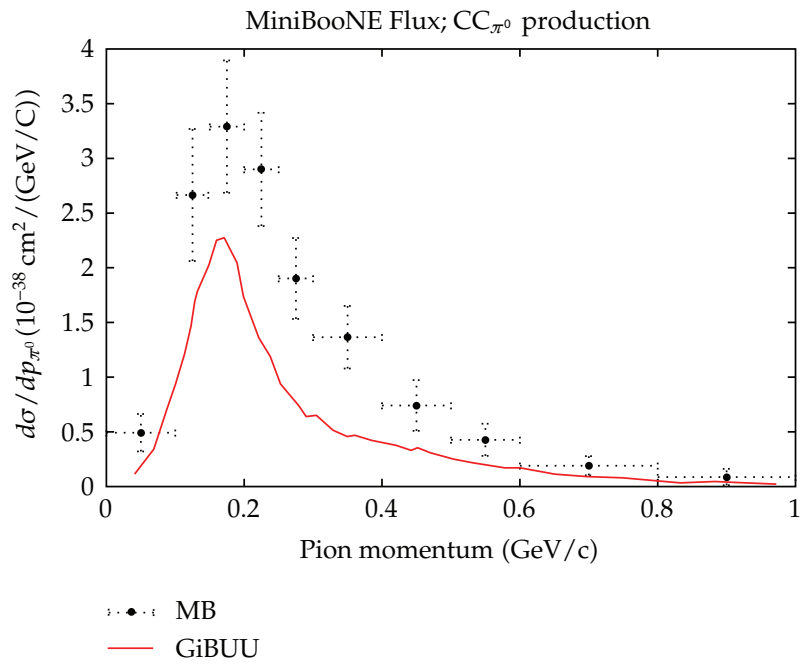

(b)

Figure 5: (a) Differential cross section for $\mathrm{CC} 1 \pi^{+}$production in the final state (all the FSI effects are included). MiniBooNE measurement [77] is compared to GiBUU [78] and NuWro computations. (b) The same for CC $\pi^{0}$ production, but only GIBUU results are shown.

\subsection{3. $\mathrm{CC} \pi^{0}$}

MiniBooNE measured also CC $1 \pi^{0}$ production cross sections. As before, the signal is defined as exactly one $\pi^{0}$ in the final state [79]. Various differential distributions are available. There is a dramatic discrepancy between the measured CC $1 \pi^{0}$ production cross section as a function of neutrino energy and NUANCE MC predictions in the region of lower energies. On average the data is larger by $56 \pm 20 \%$, but for $E_{v}<1 \mathrm{GeV}$ the disagreement is as large as a factor of 2 . In Figure 5(b) GiBUU predictions for $\mathrm{CC} \pi^{+}$are compared to the MiniBooNE data. 


\subsubsection{Ratio $\sigma\left(C C 1 \pi^{+}\right) / \sigma(C C Q E)$}

Another useful MiniBooNE measurement was the ratio $\sigma\left(\mathrm{CC} 1 \pi^{+}\right) / \sigma(\mathrm{CCQE})$ [80]. The ratio of $\mathrm{CC} 1 \pi^{+}$-like (one pion in the final state) to CCQE-like cross sections on $\mathrm{CH}_{2}$ as a function of neutrino energy was measured with an accuracy of $~ 10 \%$ in bins with highest statistics. This measurement puts constraints on the theoretical models which include QE, $\Delta$ excitation, and MEC $/ 2 \mathrm{p} 2 \mathrm{~h}$ dynamics. But still, in order to compare with theoretical model predictions to these data, FSI effects must be included. In order to make such a comparison easier, MiniBooNE provided also FSI-corrected data representing the ratio of CC1 $\pi^{+} / \mathrm{CCQE}$ cross sections at the primary interaction. The corrected results are biased by MC assumptions and in particular they neglect most of the MEC $/ 2 \mathrm{p} 2 \mathrm{~h}$ contributions which is contained in the QElike sample of events. Finally, MiniBooNE rescaled their results in order to get data points for an isoscalar target and enable comparison to old ANL and also more recent K2K data [81]. $\mathrm{K} 2 \mathrm{~K}$ measured ratio of cross sections on bound nucleons inside the nucleus corrected for FSI effects. CC $1 \pi^{+}$events were not identified on an event-by-event basis.

\subsubsection{Phenomenological Considerations}

Due to nuclear effects a comparison to the new data is possible only for MC event generators, sophisticated computation tools like GiBUU and also a few theoretical groups which are able to evaluate FSI effects.

Most of the interesting work was done within GiBUU. It turned out to be very difficult reproduce the MiniBooNE CC $1 \pi^{+}$and $\mathrm{CC} 1 \pi^{0}$ results: the measured cross section is much larger than theoretical computations. In the case of $\mathrm{CC} 1 \pi^{+}$production the discrepancy is as large as $100 \%$. It was also noted that the reported shape of the distribution of $\pi^{+}$kinetic energies is different from theoretical calculations and does not show a strong decrease at $T_{\pi^{+}}>120 \mathrm{MeV}$ located in the region of maximal probability for pion absorption.

The authors of [78] mention three possible reasons for the data/GiBUU predictions discrepancy: (i) the fact that $\Delta$ excitation axial form factor was chosen to agree with the ANL data only, neglecting the larger cross section BNL measurements; (ii) hypothetical 2p2h$1 \pi$ pion production contribution analogous to $2 \mathrm{p} 2 \mathrm{~h}$ discussed in the Section 3.3; (iii) flux underestimation in the MiniBooNE experiment. For the last point, the argument gets support from the better data/theory agreement found for the ratio, as discussed below.

In the case of $\mathrm{NC} \pi^{0}$ production, a systematical comparison was done with NuWro MC predictions with an updated FSI model for pions [64]. The overall agreement is satisfactory. Shapes of the distributions of final state $\pi^{0 \prime}$ s are affected by an interplay between pion FSI such as absorption and formation time effects, understood here as an effect of a finite $\Delta$ lifetime. It is argued that $\mathrm{NC} \pi^{0}$ production data can be very useful for benchmarking neutrino $\mathrm{MC}$ event generators.

Because of the apparent data/MC normalization discrepancy for the $\mathrm{CC} \pi^{+}$production, the interesting data is that for the ratio $\sigma\left(\mathrm{CC} 1 \pi^{+}\right.$-like) $/ \sigma$ (CCQE-like). This observable is free from the overall flux normalization uncertainty. However, it is not a direct observable quantity because in the experimental analysis it is necessary to reconstruct the neutrino energy and the procedures applied for the denominator and numerator are different. Three theoretical predictions for the ratio were published. The Giessen group compared to the MiniBooNE ratio data using the model described in [82] with the FSI effects modeled by the GiBUU code [83]. There is a significant discrepancy between the model and the data 
points: the calculated ratio is smaller. For the $\mathrm{K} 2 \mathrm{~K}$ data, the GiBUU model computations are consistent with the experimental results.

The $\sigma\left(\mathrm{CC} 1 \pi^{+}\right) / \sigma(\mathrm{CCQE})$ ratio was also analyzed in [84]. In this analysis many nuclear effects were included: the in medium $\Delta$ self-energy (both real and imaginary parts), FSI effects within the cascade model of [85], RPA corrections for the CCQE. ... Computations did not include contributions from the nonresonant background and from higher resonances. The contribution from the coherent pion production evaluated with the model of [86] (about $5 \%$ of the $\pi^{+}$signal, a surprisingly large fraction) was also included in computations. The model predictions agree with MiniBooNE measurement for $E_{v}<1 \mathrm{GeV}$ and are below MiniBooNE data for larger neutrino energies.

Finally, NuWro MC results for the ratio given in [87] are slightly below the data points for larger neutrino energies.

\subsection{Theoretical Analyses}

It has been known since ANL and BNL pion production measurements that although being a dominant mechanism, $\Delta$ excitation alone cannot reproduce the data and that nonresonant background terms must be included in the theoretical models. There were many attempts in the past to develop suitable models but usually they were not very well justified from the theoretical point of view.

\subsubsection{Nonresonant Background}

A general scheme to analyze weak pion production in the $\Delta$ region based on the chiral symmetry was proposed a few years ago in [88]. The model is supposed to work well in the kinematical region $W<1.3-1.4 \mathrm{GeV}$ that is, in the $\Delta$ region. The background contribution is particularly important at the pion production threshold, for values of $W$ near $M+m_{\pi}$. Vector form factors are taken from the electroproduction data and fits to helicity amplitudes [89]. Although particulary important for the channels $v_{\ell} n \rightarrow \ell^{-} p \pi^{0}$ and $v_{\ell} n \rightarrow \ell^{-} n \pi^{+}$the background terms contribute also to the channel $v_{\ell} p \rightarrow \ell^{-} p \pi^{+}$changing the fitted values of the nucleon- $\Delta$ transition matrix elements. A comparison to existing NC pion production data was done as well and a good agreement was also found. An interesting question raised by the authors of [88] is that of unitarity. Their approach does not satisfy requirements of the Watson theorem and this can have some consequences for example, worse agreement with the antineutrino pion production data.

The model of the nonresonant background was used by the Giessen group which made several qualitative comparisons to both the ANL and BNL pion production data in the region $W<1.4 \mathrm{GeV}$ neglecting deuterium effects [90]. In the case of neutron channels the model predictions are much below the BNL data points and this is because the axial form factor parameters were optimized to the ANL data only. This choice goes back to the paper [89] where the authors came to the conclusion that the ANL and BNL data for the $\Delta^{++}$excitation are not compatible.

\subsubsection{Reanalysis of Old Bubble Chamber Data}

The issue of nucleon- $\Delta$ transition matrix element was discussed also in other papers. The questions are what is the value of the $C_{5}^{A}(0)$ ? How relevant are deuterium nuclear effects in dealing with ANL and BNL data? How much tension is there between both data samples? 
In [88] a fit was done to the ANL data in the $\Delta^{++}$channel only with the results: $C_{5}^{A}(0)=$ $0.867 \pm 0.075$ and $M_{A \Delta}=0.985 \pm 0.082 \mathrm{GeV}$. The obtained value of $C_{5}^{A}(0)$ was very different from what follows from off-diagonal Goldberger-Treiman relations $\left(C_{5}^{A}(0) \approx 1.15\right)$.

The authors of [87] made a fit to both ANL and BNL data including in the $x^{2}$, terms with the overall flux normalization uncertainties, separate for ANL and BNL. In the fit the deuterium nuclear effects were included as correction factors to the $Q^{2}$ distributions of events, using the results of [91]. The main conclusion was that ANL and BNL data are in fact consistent. This statement was verified in a rigorous way using parameter goodness of fit method [92]. In the dipole parameterization of the $C_{5}^{A}\left(Q^{2}\right)$ form factor the best fit values were found to be $C_{5}^{A}(0)=1.14 \pm 0.08$ and $M_{A}=0.95 \pm 0.04$. Only $\Delta^{++}$channel was analyzed and like in [75] non-resonant background contributions was not included.

So far the most complete analysis of both ANL and BNL data was performed in [93]: a nonresonant background was included and also deuterium effects were taken into account in the systematic way. The authors made several fits with various assumptions and in the fit IV they obtained $C_{A}^{5}(0)=1.00 \pm 0.11$.

\subsubsection{Other Theoretical Approaches}

In [94] the dynamical pion cloud effects are imposed on bare quark $N-\Delta$ transition matrix elements. The model is able to reproduce both ANL and BNL weak pion production data.

The authors of [95] focus on the consistent use of the $\Delta$ propagator. They show that the computations relying on the standard Rarita-Schwinger propagator could lead to an underestimation of the weak pion production cross section.

\subsection{Coherent Pion Production}

In coherent pion production $(\mathrm{COH})$ the target nucleus remains in the ground state. There are four possible channels, for CC and NC reactions, neutrinos, and antineutrinos. A clear experimental signal for the $\mathrm{COH}$ reaction for high energies was observed and the aim of recent measurements was to fill a gap in the knowledge of a region around $\sim 1 \mathrm{GeV} \mathrm{COH}$ cross-sections. At larger neutrino energies a recent measurement was done by MINOS which reported a NC reaction cross section at $\left\langle E_{v}\right\rangle=4.9 \mathrm{GeV}$ to be consistent with the predictions of the Berger-Sehgal model (see below).

\subsubsection{Experimental Results}

In the case of the NC reaction, MiniBooNE [73] and SciBooNE [96] searched for the COH component. SciBooNE [96] evaluated the ratio of the $\mathrm{COH} \mathrm{NC} \pi^{0}$ production to the total CC cross section as $(1.16 \pm 0.24) \%$.

For the NC reaction MiniBooNE evaluated the $\mathrm{COH}$ component (plus possible hydrogen diffractive contribution about which little is known) in the NC $\pi^{0}$ production as $19.5 \%\left(\right.$ at $\left.\left\langle E_{v}\right\rangle \sim 1 \mathrm{GeV}\right)$ and then the overall flux averaged overall $\mathrm{NC} 1 \pi^{0}$ cross section as $(4.76 \pm 0.05 \pm 0.76) \cdot 10^{-40} \mathrm{~cm}^{2} /$ nucleon. Unfortunately, it is difficult to translate both measurements into the absolutely normalized value of the $\mathrm{NC} \mathrm{COH}$ cross section because of strong dependence on the NUANCE MC generator used in the data analysis. In NUANCE, $\mathrm{RES}, \mathrm{COH}$, and BGR (nonresonance background) $\mathrm{NC} \pi^{0}$ reactions are defined according to primary interaction and $\mathrm{COH}$ pions are also subject to FSI. In the MiniBooNE analysis the fit 
is done for the composition of the sample of $\mathrm{NC} \pi^{0}$ events in terms of three components, and the $\mathrm{COH}$ fraction is defined as $x_{\mathrm{COH}} /\left(x_{\mathrm{COH}}+x_{\mathrm{RES}}\right)$.

In the case of the CC reaction, K2K [97] and SciBooNE [98] reported no evidence for the $\mathrm{COH}$ component. For the K2K analysis, the $90 \%$ confidence limit upper bound for the $\mathrm{COH}$ cross sections on carbon was estimated to be $0.6 \%$ of the inclusive $\mathrm{CC}$ cross section. The SciBooNE upper limits (also for the carbon target) are $0.67 \%$ at $\left\langle E_{v}\right\rangle \sim 1.1 \mathrm{GeV}$, and $1.36 \%$ at $\left\langle E_{v}\right\rangle \sim 2.2 \mathrm{GeV}$. SciBooNE reported also the measurement of the ratio of $\mathrm{CC} \mathrm{COH} \pi^{+}$to $\mathrm{NC}$ $\mathrm{COH} \pi^{0}$ production and estimated it as $0.14_{-0.28}^{+0.30}$. This is a surprisingly low value, which disagrees with results from the theoretical models which at SciBooNE energies typically predict values somehow smaller 2. For massless charged leptons isospin symmetry implies the value of 2 for this ratio and the finite mass corrections make the predicted ratio smaller.

\subsubsection{Theoretical Developments}

Higher neutrino energy $\left(\left\langle E_{v}\right\rangle \geq 2 \mathrm{GeV}\right) \mathrm{COH}$ production data (including recent NOMAD measurement) were successfully explained with a PCAC based model [99]. Adler's theorem relates $\sigma_{\mathrm{COH}}\left(v+X \rightarrow v+X+\pi^{0}\right)$ at $Q^{2} \rightarrow 0$ to $\sigma\left(\pi^{0}+X \rightarrow \pi^{0}+X\right)$. Subsequently, the model for the CC reaction, has been upgraded [100] to include lepton mass effects important for low $E_{v}$ studies. The new model predicts the $\sigma_{\mathrm{COH}}\left(\pi^{+}\right) / \sigma_{\mathrm{COH}}\left(\pi^{0}\right)$ ratio at $E_{v}=1 \mathrm{GeV}$ to be 1.45 rather than 2. Another important improvement was to use a better model for $d \sigma\left(\pi+{ }^{12} \mathrm{C} \rightarrow\right.$ $\pi+{ }^{12} \mathrm{C}$ ) / $d t$ in the region of pion kinematical energy $100 \mathrm{MeV}<T_{\pi}<900 \mathrm{MeV}$. As a result, the predicted $\mathrm{COH}$ cross section from the model became reduced by a factor of 2-3 [101]. The PCAC-based approach was also discussed in [102] and critically rederived in [103].

At lower energies the microscopic $\Delta$ dominance models for the $\mathrm{COH}$ reaction [104108] are believed to be more reliable. Within microscopic models there are still various approaches, for example, due to differences in the treatment of the nonresonant background. The absolute normalization of the predicted cross section depends on the adopted value of the $N \rightarrow \Delta$ form factor $\mathrm{C}_{5}^{A}(0)$ because $\sigma_{\mathrm{COH}} \sim\left(\mathrm{C}_{5}^{A}(0)\right)^{2}$.

\subsection{Generators}

Almost all MC events generators rely on the old Rein-Sehgal resonance model for pion resonance production [109]. The model is based on the quark resonance model and includes contributions from 18 resonances covering the region $W<2 \mathrm{GeV}$. The model is easily implementable in MC generators and it has only one set of vector and axial form factors. In the original model, the charged lepton is assumed to be massless and prescriptions to cope with this problem were proposed in [100,110]. It was also realized that the RS model can be improved in the $\Delta$ region by modifying both vector and axial form factors using either old deuterium or new MiniBooNE pion production data [21, 111, 112].

As for coherent pion production, all the MCs use the Rein-Sehgal $\mathrm{COH}$ model [99]. The analysis of MC event generators and theoretical models done in [22] show that in the $1-2 \mathrm{GeV}$ energy region, the Rein Sehgal $\mathrm{COH}$ model predictions disagree significantly with all the recent theoretical computations and experimental results.

A crucial element of MC is the FSI model. These are typically semiclassical intranuclear cascade models. The topic of FSI goes far beyond the scope of this paper and we only note that the progress in understanding the experimental data requires more reliable FSI models. The existing models should be systematically benchmarked with electro- and photoproduction data as it was done in the case of GIBUU. 


\subsection{Duality}

Bridging the region between RES and DIS (where with a good approximation interactions occur on quarks) dynamics is a practical problem which must be resolved in all MC event generators. In MC event generators "DIS" is defined as "anything but QE and RES," what is usually expressed as a condition on the invariant hadronic mass of the type $W>1.6 \mathrm{GeV}$ or $W>2 \mathrm{GeV}$ or so. Notice however that such a definition of "DIS" contains a contribution from the kinematical region $Q^{2}<1 \mathrm{GeV}^{2}$ which is beyond the applicability of the genuine DIS formalism. RES/DIS transition region is not only a matter of an arbitrary choice but is closely connected with the hypothesis of quark-hadron duality.

Investigation of structure functions introduced in the formalism of the inclusive electron-nucleon scattering led Bloom and Gilman to the observation that the average over resonances is approximately equal to the leading twist contribution measured in the completely different DIS region. One can distinguish two aspects of duality: (i) resonant structure functions oscillate around a DIS scaling curve; (ii) the resonant structure functions for varying values of $Q^{2}$ slide along the DIS curve evaluated at fixed $Q_{\mathrm{DIS}}^{2}$.

In order to quantify the degree in which the duality is satisfied one defines the ratio of integrals over structure functions from RES and DIS:

$$
\mathcal{R}\left(Q^{2}, Q_{\mathrm{DIS}}^{2}\right)=\frac{\int_{\xi_{\min }}^{\xi_{\max }} d \xi F_{j}\left(\xi, Q^{2}\right)}{\int_{\xi_{\min }}^{\xi_{\max }} d \xi F_{j}\left(\xi, Q_{\mathrm{DIS}}^{2}\right)} .
$$

The integrals are in the Nachtmann variable $\xi\left(x, Q^{2}\right)=2 x /\left(1+\sqrt{1+4 x^{2} M^{2} / Q^{2}}\right)$ and the integration region is defined as $W_{\min }<W<W_{\max }$. Typically $W_{\min }=M+m_{\pi}$ and $W_{\max }=$ $1.6, \ldots, 2.0 \mathrm{GeV}$. In the case of DIS, the value of $Q_{\mathrm{DIS}}^{2}$ is much larger and as a consequence the integral over $\xi$ runs over a quite different region in $W$.

Neutrino-nucleon scattering duality studies are theoretical in their nature because the precise data in the resonance region are still missing. The duality was studied in three papers: $[94,113,114]$. For neutrino interactions the duality can be satisfied only for the isospin average target. This is because the RES structure functions for proton are much larger than for neutron and in the case of DIS structure functions the situation is opposite.

Theoretical studies were done with a model which contains resonances from the first and second resonance region but not the background contribution and with the Rein-Sehgal model which is commonly used in $\mathrm{MC}$ event generators. If the resonance region is confined to $W<1.6 \mathrm{GeV}$ the duality as defined in (4.1) is satisfied at the $75-80 \%$ level. If the resonance region is extended to $W<2 \mathrm{GeV}$ the value of the integral in (4.1) is only about $50 \%$. These results are to some extent model dependent but a general tendency is that for larger $W$, DIS structure functions are much larger than the resonance contribution, as clearly seen from Figure 3 in [113] and Figure 7 in [114]. As shown in [114] there is also a 5\% uncertainty coming from an arbitrary choice of $Q_{\text {DIS }}^{2}$.

Two component duality hypothesis states that resonance contribution is dual to the valence quarks and the nonresonant background to the sea. Investigation done within the Rein Sehgal model with $W<2 \mathrm{GeV}$ revealed no signature of two component duality. Quarkhadron duality was also investigated in the case of neutrino nucleus interactions [115].

As a practical procedure for addressing this region, Bodek and Yang [116] have introduced and refined a model that is used by many contemporary neutrino event generators such as NEUGEN and its successor GENIE to bridge the kinematic region between 
the Delta and full DIS. The model has been developed for both neutrino- and electronnucleon inelastic scattering cross sections using leading order parton distribution functions and introducing a new scaling variable they call $\xi_{w}$.

Nonperturbative effects that are prevalent in the kinematic region bridging the resonance and DIS regimes are described using the $\xi_{w}$ scaling variable, in combination with multiplicative $K$ factors at low $Q^{2}$. The model is successful in describing inelastic charged lepton-nucleon scattering, including resonance production, from high-to-low $Q^{2}$. In particular, the model describes existing inelastic neutrino-nucleon scattering measurements.

Their proposed scaling variable, $\xi_{w}$ is derived using energy momentum conservation and assumptions about the initial/final quark mass and $P_{T}$. Parameters are built into the derivation of $\xi_{w}$ to account (on average) for the higher order QCD terms and dynamic higher twist that is covered by an enhanced target mass term.

At the juncture with the DIS region, the Bodek-Yang model incorporates the GRV98 [117] LO parton distribution functions replacing the variable $x$ with $\xi_{w}$. They introduce " $K$ factors", different for sea and valence quarks, to multiply the PDFs so that they are correct at the low $Q^{2}$ photo-production limit. A possible criticism of the model is the requirement of using the rather dated GRV98 parton distribution functions in the DIS region so the bridge to the lower $W$ kinematic region is seamless.

\section{5. v-A Deep-Inelastic Scattering: Introduction}

Although deep-inelastic scattering (DIS) is normally considered to be a topic for much higher energy neutrinos, wide-band beams such as the Fermilab NuMI and the planned LBNE beams do have real contributions from DIS that are particularly important in feed down to the background that must be carefully considered. In addition, there are $x$-dependent nuclear effects that should be taken into account when comparing results from detectors with different nuclei and even when comparing results from "identical" near and far detectors when the neutrino spectra entering the near and far detectors are different.

For this paper, the definition of deep-inelastic scattering (DIS) is the kinematic-based definition with $W \geq 2.0 \mathrm{GeV}$ and $Q^{2} \geq 1.0 \mathrm{GeV}$. This is mostly out of the resonance production region and allows a fit to parton distribution functions. As said in Introduction, this is unfortunately not the definition used by several modern Monte Carlo generators that do not differentiate between simply "inelastic" interactions and deep-inelastic interactions calling everything beyond the Delta simply DIS. This is an unfortunate confusing use of nomenclature by the generators.

In general, deep-inelastic scattering offers an opportunity to probe the partonic structure of the nucleon both in its free state and when the nucleon is bound in a nucleus. Description of the partonic structure can include parton distribution functions (PDFs) giving the longitudinal, transverse, and spin distributions of quarks within the nucleon as well as, for example, the hadron formation zone giving the time/length it takes for a struck quark to fully hadronize into a strong-interacting hadron.

Neutrino scattering can play an important role in extraction of these fundamental parton distribution functions (PDFs) since only neutrinos via the weak interaction can resolve the flavor of the nucleon's constituents: $v$ interacts with $d, s, \bar{u}$ and $\bar{c}$ while the $\bar{v}$ interacts with $u, c, \bar{d}$ and $\bar{s}$. The weak current's unique ability to "taste" only particular quark flavors significantly enhances the study of parton distribution functions. High-statistics measurement of the nucleon's partonic structure, using neutrinos, could complement studies with electromagnetic probes. 
In the pursuit of precision measurements of neutrino oscillation parameters, large data samples and dedicated effort to minimize systematic errors could allow neutrino experiments to independently isolate all SIX of the weak structure functions $F_{1}^{\nu N}\left(x, Q^{2}\right), F_{1}^{\bar{\nu} N}\left(x, Q^{2}\right), F_{2}^{\nu N}\left(x, Q^{2}\right), F_{2}^{\bar{\nu} N}\left(x, Q^{2}\right), x F_{3}^{\nu N}\left(x, Q^{2}\right)$, and $x F_{3}^{\bar{\nu} N}\left(x, Q^{2}\right)$ for the first time. Then, by taking differences and sums of these structure functions, specific parton distribution functions in a given $\left(x, Q^{2}\right)$ bin can in turn be better isolated. Extracting this full set of structure functions will rely on the $y$-variation of the structure function coefficients in the expression for the cross-section. In the helicity representation, for example:

$$
\begin{aligned}
\frac{d^{2} \sigma^{v}}{d x d Q^{2}}=\frac{G_{F}^{2}}{2 \pi x}[ & \frac{1}{2}\left(F_{2}^{v}\left(x, Q^{2}\right)+x F_{3}^{v}\left(x, Q^{2}\right)\right) \\
& \left.+\frac{(1-y)^{2}}{2}\left(F_{2}^{v}\left(x, Q^{2}\right)-x F_{3}^{v}\left(x, Q^{2}\right)\right)-2 y^{2} F_{L}^{v}\left(x, Q^{2}\right)\right],
\end{aligned}
$$

where $F_{L}$ is the longitudinal structure function representing the absorption of longitudinally polarized Intermediate Vector Boson.

By analyzing the data as a function of $(1-y)^{2}$ in a given $\left(x, Q^{2}\right)$ bin for both $v$ and $\bar{v}$, all six structure functions could be extracted.

Somewhat less demanding in statistics and control of systematics, the "average" structure functions $F_{2}\left(x, Q^{2}\right)$ and $x F_{3}\left(x, Q^{2}\right)$ can be determined from fits to combinations of the neutrino and antineutrino differential cross sections and several assumptions. The sum of the $v$ and $\bar{v}$ differential cross sections, yielding $F_{2}$ then can be expressed as follows:

$$
\frac{d^{2} \sigma^{v}}{d x d y}+\frac{d^{2} \sigma^{\bar{v}}}{d x d y}=\frac{G_{F}^{2} M E}{\pi}\left[2\left(1-y-\frac{M x y}{2 E}+\frac{y^{2}}{2} \frac{1+4 M^{2} x^{2} / Q^{2}}{1+R_{L}}\right) F_{2}+y\left(1-\frac{y}{2}\right) \Delta x F_{3}\right],
$$

where $R_{L}$ is equal to $\sigma_{L} / \sigma_{T}$ and now $F_{2}$ is the average of $F_{2}^{v}$ and $F_{2}^{\bar{v}}$ and the last term is proportional to the difference in $x F_{3}$ for neutrino and antineutrino probes, $\Delta x F_{3}=x F_{3}^{v}-x F_{3}^{\bar{v}}$. In terms of the strange and charm parton distribution function $s$ and $c$, at leading order, assuming symmetric $s$ and $c$ seas, this is $4 x(s-c)$.

The cross sections are also corrected for the excess of neutrons over protons in the target (e.g., the Fe correction is $5.67 \%$ ) so that the presented structure functions are for an isoscalar target. A significant step in the determination of $F_{2}\left(x, Q^{2}\right)$ in this manner that affects the low- $x$ values is the assumed $\Delta x F_{3}$ and $R_{L}\left(x, Q^{2}\right)$. Recent analyses use, for example, a NLO QCD model as input (TRVFS $[118,119])$ and assumes an input value of $R_{L}\left(x, Q^{2}\right)$ that comes from a fit to the world's charged-lepton measurements [120]. This could be an additional problem since, as will be suggested, $R_{L}\left(x, Q^{2}\right)$ can be different for neutrino as opposed to charged-lepton scattering.

The structure function $x F_{3}$ can be determined in a similar manner by taking the difference in $v$ and $\bar{v}$ differential cross sections. 


\subsection{The Physics of Deep-Inelastic Scattering}

There have been very few recent developments in the theory of deep-inelastic scattering. The theory has been well established for years. The most recent developments in neutrino DIS scattering involve the experimental determination of parton distribution functions of nucleons within a nucleus, so-called nuclear parton distribution functions (nPDF). The more contemporary study of $v$ nucleus deep-inelastic scattering using high-statistics experimental results with careful attention to multiple systematic errors began with the CDHSW, $\mathrm{CCFR} / \mathrm{NuTeV} v \mathrm{Fe}$, the NOMAD $v \mathrm{C}$, and the CHORUS $v \mathrm{~Pb}$ experiments. Whereas $\mathrm{NuTeV}$ [121] and CHORUS [122] Collaborations have published their full data sets, NOMAD [123] has not yet done so. This short summary of DIS physics will concentrate on nuclear/nucleon parton distribution functions.

\subsubsection{Low-and-High $Q^{2}$ Structure Functions: Longitudinal and Transverse}

Since the current and future neutrino beams designed for neutrino oscillation experiments will be concentrating on lower energy neutrinos $(1-5 \mathrm{GeV})$, many of the interactions will be at the lower- $Q$ edge of DIS or even in the "soft" DIS region, namely, $W \geq 2.0 \mathrm{GeV}$ however, with $Q^{2} \leq 1.0 \mathrm{GeV}^{2}$. Understanding the physics of this kinematic region is therefore important.

Since both the vector and axial-vector part of the transverse structure function $F_{T}$ go to 0 at $Q^{2}=0$ (similar to $\ell^{ \pm}$charged-lepton vector current scattering), the low- $Q^{2}$ region $v$ and $\bar{v}$ cross sections are dominated by the longitudinal structure function $F_{L}$. The longitudinal structure function is composed of a vector and axial-vector component $F_{L}^{\mathrm{VC}}$ and $F_{L}^{\mathrm{AC}}$ and the low- $Q^{2}$ behavior of these components is not the same as in the transverse case. The conservation of the vector current (CVC) suggests that $F_{L}^{\mathrm{VC}}$ behaves as the vector current in charged-lepton scattering and vanishes at low $Q^{2}$. However, the axial-vector current is not conserved and is related to the pion field via PCAC, so there is a surviving low $Q^{2}$ contribution from this component [124] and $F_{L}^{\mathrm{AC}}$ dominates the low $Q^{2}$ behavior. Consequently, the ratio $R=F_{L} / F_{T}$ is divergent for neutrino interactions. This is substantially different from the scattering of charged leptons for which $R$ is vanishing as $Q^{2}$ and using measurement of $R$ from charged lepton scattering to determine $F_{2}$ for neutrino scattering is obviously wrong for lower $Q$. In addition, this non-vanishing and dominant longitudinal structure function could be important for the interpretation of low- $Q^{2}$ nuclear effects with neutrinos to be described shortly.

\subsubsection{Low-and-High $Q^{2}$ Structure Functions: $1 / Q^{2}$ Corrections}

Using a notation similar to that of [125], the total structure function can be expressed in a phenomenological form:

$$
F_{i}\left(x, Q^{2}\right)=F_{i}^{\mathrm{LT}}\left(x, Q^{2}\right)\left(1+\frac{C_{4}(x)}{Q^{2}}\right),
$$

where $i=1,2,3$ refers to the type of the structure function. Using $i=2$ as an example, then $F_{2}^{\mathrm{LT}}$ is the leading twist component that has already included target mass corrections (TMC) and $C_{4}$ is the coefficient of the twist- 4 term, the first higher-twist term proportional to $\left(1 / Q^{2}\right)$. There are, of course, further higher-twist terms $H_{i}^{(T=6)}(x) / Q^{4}+\cdots$ proportional to ever increasing powers of $1 / Q^{2}$ however, for most phenomenological fits, the dominant leading twist plus twist- 4 term are sufficient to describe the data. The target mass corrections are kinematic in origin and involve terms suppressed by powers of $M^{2} / Q^{2}$ while the higher 
twist terms are dynamical in origin and are suppressed as mentioned by powers of $1 / Q^{2}$. These higher-twist terms are associated with multiquark or quark and gluon fields and it is difficult to evaluate their magnitude and shape from first principles. As with the kinematic target mass corrections, these must be taken into account in analyses of data at low $Q^{2}$ and especially at large $x$. At higher $Q^{2}$ the contribution of the HT terms is negligible and there are various global fits $[126,127]$ to the structure functions (among various scattering input) to determine the parton distribution functions (PDFs) that do not include any HT terms.

The analysis of nuclear PDFs to be described shortly uses data from a TeVatron neutrino experiment at very high neutrino energies and thus is one of the analyses that does not need to be concerned with higher-twist corrections. However, the current neutrinooscillation oriented beam-lines are not high-energy and the analyses of this data may indeed need to consider both target mass corrections and higher-twist. If indeed inclusion of highertwist in these analyses becomes necessary, the authors of [125] stress the importance of explicitly including both the target mass corrections and the higher twist corrections, even though they have very different physical origin and can have very different $x$ dependence. It is important to note, as mentioned, that there are both nucleon and nuclear PDFs depending on the target. The relation between them, called nuclear correction factors, are currently being studied for both $v-A$ and $\ell^{ \pm} A$. There are early indications that the nuclear correction factors for these two processes may not be the same.

\section{Recent DIS Measurements: Neutrino Iron Scattering Results}

The difficulty, of course, is that modern neutrino oscillation experiments demand high statistics which means that the neutrinos need massive nuclear targets to acquire these statistics. This, in turn, complicates the extraction of free nucleon PDFs and demands nuclear correction factors that scale the results on a massive target to the corresponding result on a nucleon target. The results of the latest study of QCD using neutrino scattering comes from the $\mathrm{NuTeV}$ experiment [121]. The NuTeV experiment was a direct followup of the CCFR experiment using nearly the same detector as CCFR but with a different neutrino beam. The NuTeV experiment accumulated over 3 million $v$ and $\bar{v}$ events in the energy range of 20 to $400 \mathrm{GeV}$ off a manly Fe target. A comparison of the NuTeV results with those of CCFR and the predictions of the major PDF-fitting collaborations (CTEQ and MRST $[126,127]$ ) is shown in Figure 6.

The main points are that the NuTeV $F_{2}$ agrees with CCFR for values of $x_{B j} \leq 0.4$ but is systematically higher for larger values of $x_{B j}$ culminating at $x_{B j}=0.65$ where the NuTeV result is $20 \%$ higher than the CCFR result. NuTeV agrees with charged lepton data for $x_{B j} \leq$ 0.5 but there is increasing disagreement for higher values. Although $\mathrm{NuTeV} F_{2}$ and $x F_{3}$ agree with theory for medium $x$, they find a different $Q^{2}$ behavior at small $\mathrm{x}$ and are systematically higher than theory at high $x$. These results can be summarized in four main questions to ask subsequent neutrino experiments.

(i) At high $x$, what is the behavior of the valence quarks as $x \rightarrow 1.0$ ?

(ii) At all $x$ and $Q^{2}$, what is yet to be learned if we can measure all six $v$ and $\bar{v}$ structure functions to yield maximal information on the parton distribution functions?

(iii) At all $x$, how do nuclear effects with incoming neutrinos differ from nuclear effects with incoming charged leptons? 


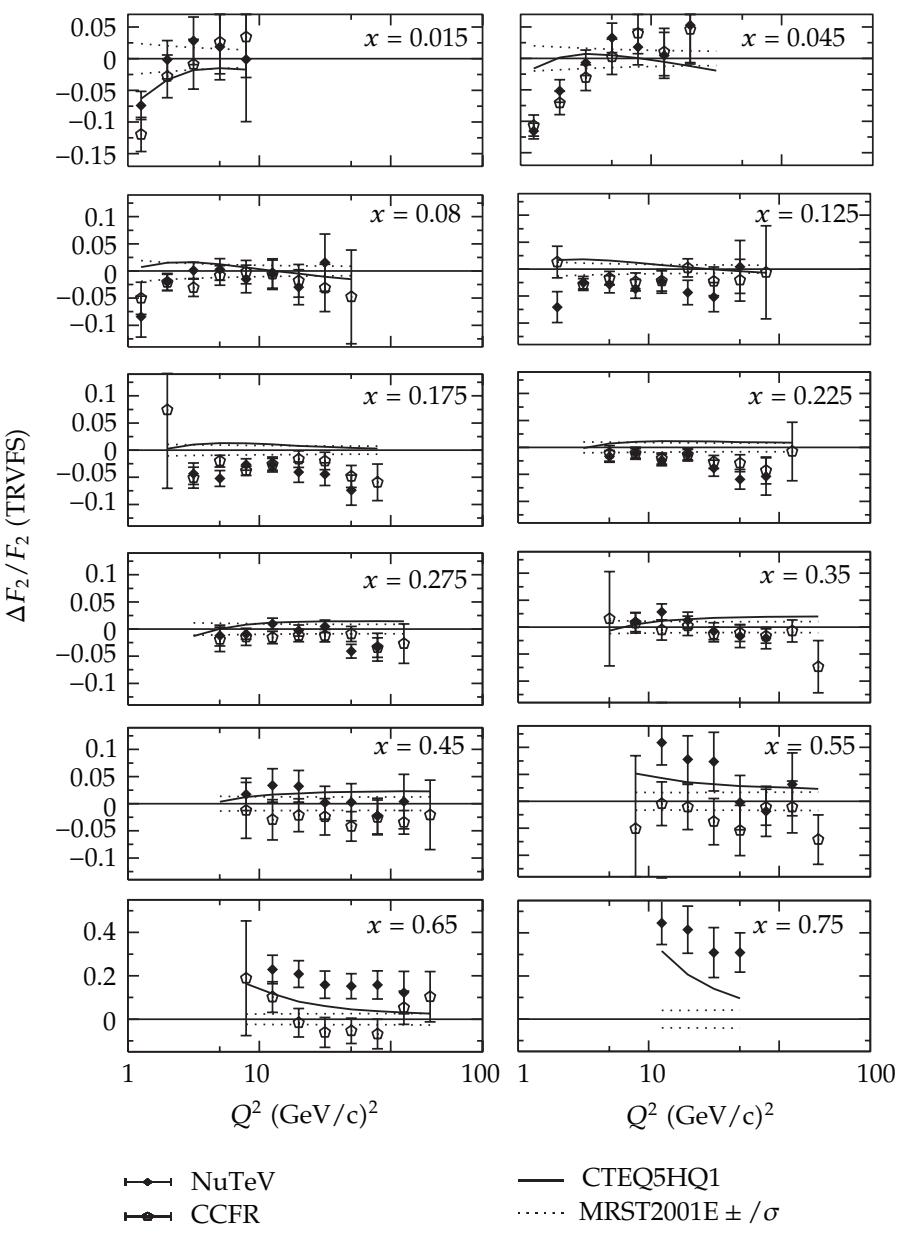

Figure 6: A comparison of the measurements of the $F_{2}$ structure function by NuTeV and CCFR and the predictions from the global PDF fits of the MRST and CTEQ collaboration [128] that does not use the $\mathrm{NuTeV}$ data points as input to their fit. The model predictions have already been corrected for target mass and, most significantly, nuclear effects assuming these corrections are the same for charge-lepton and neutrino interactions.

This last item highlights an overriding question when trying to get a global view of structure functions from both neutrino and charged-lepton scattering data. How do we compare data off nuclear targets with data off nucleons and, the associated question, how do we scale nuclear target data to the comparable nucleon data. In most PDF analyses, the nuclear correction factors were taken from $\ell^{ \pm}$-nucleus scattering and used for both chargedlepton and neutrino scattering. Recent studies by a CTEQ-Grenoble-Karlsruhe collaboration (called nCTEQ) [129] have shown that there may indeed be a difference between the chargedlepton and neutrino correction factors.

The data from the high-statistics $v$-DIS experiment, NuTeV summarized above, was used to perform a dedicated PDF fit to neutrino-iron data [130]. The methodology for this fit is parallel to that of the previous global analysis [131] but with the difference that only Fe data has been used and no nuclear corrections have been applied to the analyzed data; hence, the resulting PDFs are for a proton in an iron nucleus-nuclear parton distribution functions 


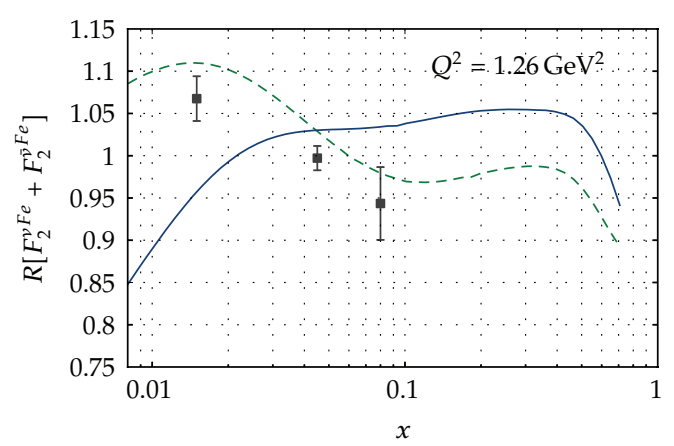

(a)

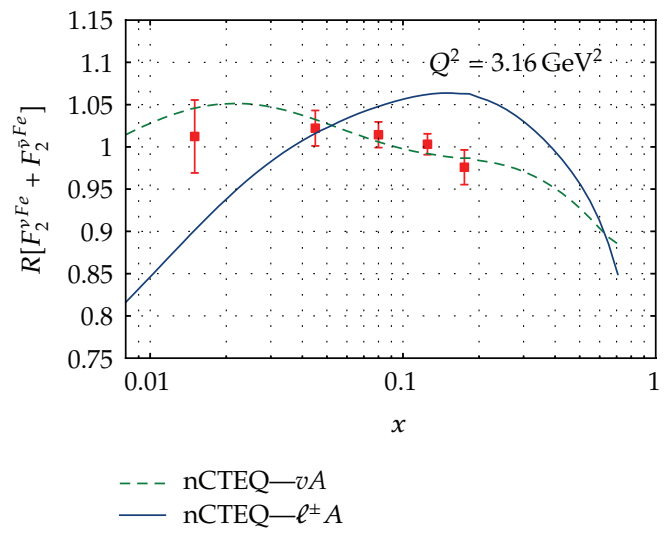

(c)

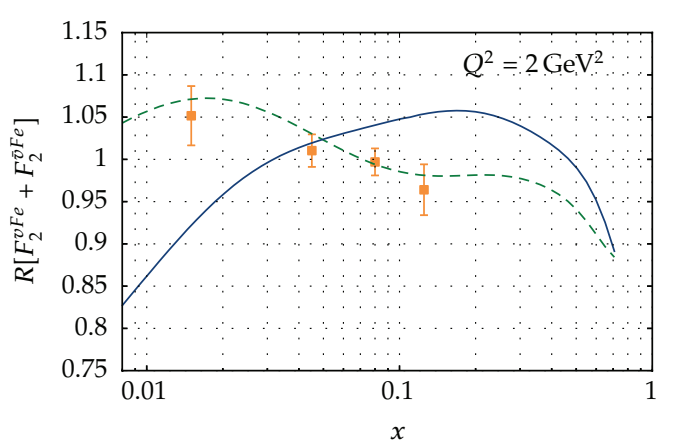

(b)

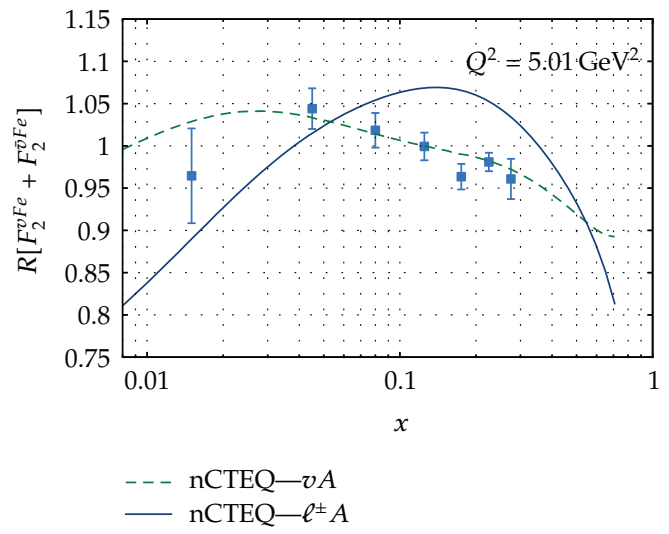

(d)

Figure 7: Nuclear correction factor $R$ for the average $F_{2}$ structure function in charged current $v$ Fe scattering at $Q^{2}=1.2,2.0,3.2$, and $5.0 \mathrm{GeV}^{2}$ compared to the measured $\mathrm{NuTeV}$ points. The green dashed curve shows the result of the nCTEQ analysis of $v A$ (CHORUS, CCFR, and NuTeV) differential cross sections plotted in terms of the average $F_{2}^{\mathrm{Fe}}$ divided by the results obtained with the reference fit (free proton) PDFs. For comparison, the nCTEQ fit to the charged-lepton data is shown by the solid blue curve.

(for more details of the fitting techniques and resulting comparisons with charged-lepton scattering see Part II of [132]).

By comparing these iron PDFs with the free-proton PDFs (appropriately scaled) a neutrino-specific heavy target nuclear correction factor $R$ can be obtained which should be applied to relate these two quantities. It is also of course possible to combine these fitted $\mathrm{nPDF}$ to form the individual values of the average of $F_{2}(v A)$ and $F_{2}(\bar{v} A)$ for a given $x, Q^{2}$ to compare directly with the NuTeV published values of this quantity. This was recently done and the nCTEQ preliminary results [133] for low- $Q^{2}$ are shown in Figure 7. Although the neutrino fit has general features in common with the charged-lepton parameterization, the magnitude of the effects and the $x$-region where they apply are quite different. The present results are noticeably flatter than the charged-lepton curves, especially at low- and moderate$x$ where the differences are significant. The comparison between the nCTEQ fit, that passes through the $\mathrm{NuTeV}$ measured points, and the charged-lepton fit is very different in the lowest- $x$, lowest- $Q^{2}$ region and gradually approaches the charged-lepton fit with increasing $Q^{2}$. However, the slope of the fit approaching the shadowing region from higher $x$ where the $\mathrm{NuTeV}$ measured points and the nCTEQ fit are consistently below the charged-lepton $A$ 
fit, make it difficult to reach the degree of shadowing evidenced in charged-lepton nucleus scattering at even higher $Q^{2}$.

The general trend is that the antishadowing region is shifted to smaller $x$ values, and any turn-over at low $x$ is minimal given the PDF uncertainties. More specifically, there is no indication of "shadowing" in the NuTeV neutrino results at low- $Q^{2}$. In general, these plots suggest that the size of the nuclear corrections extracted from the $\mathrm{NuTeV}$ data are smaller than those obtained from charged lepton scattering.

\subsection{Comparison of the $\ell^{ \pm} A$ and $v A$ Nuclear Correction Factors}

For the nCTEQ analysis, the contrast between the charged-lepton $\left(\ell^{ \pm} A\right)$ case and the neutrino $(v A)$ case is striking. While the nCTEQ fit to charged-lepton and Drell-Yan data generally align with the other charged-lepton determinations, the neutrino results clearly yield different behavior as a function of $x$, particularly in the shadowing/antishadowing region. In the $\bar{v}$ case, these differences are smaller but persist in the low- $x$ shadowing region. The nCTEQ collaboration emphasize that both the charged-lepton and neutrino results come directly from global fits to the data, there is no model involved. They further suggest that this difference between the results in charged-lepton and neutrino DIS is reflective of the long-standing "tension" between the light-target charged-lepton data and the heavy-target neutrino data in the historical global PDF fits $[134,135]$. Their latest results suggest that the tension is not only between charged-lepton light-target data and neutrino heavy-target data, but also between neutrino and charged-lepton heavy-target data. In other words a difference between charged-lepton $\left(\ell^{ \pm} A\right)$ and the neutrino $(v A)$ when comparing the same $A$.

Concentrating on this interesting difference found by the nCTEQ group, if the nuclear corrections for the $\ell^{ \pm} A$ and $v A$ processes are indeed different there are several far-reaching consequences. Considering this, the nCTEQ group has performed a unified global analysis [129] of the $\ell^{ \pm} A, D Y$, and $v A$ data (accounting for appropriate systematic and statistical errors) to determine if it is possible to obtain a "compromise" solution including both $\ell^{ \pm} A$ and $v A$ data. Using a hypothesis-testing criterion based on the $x^{2}$ distribution that can be applied to both the total $x^{2}$ as well as to the $x^{2}$ of individual data sets, they found it was not possible to accommodate the data from $v A$ and $\ell^{ \pm} A$ DIS by an acceptable combined fit.

That is, when investigating the results in detail, the tension between the $\ell^{ \pm} \mathrm{Fe}$ and $v \mathrm{Fe}$ data sets permits no possible compromise fit which adequately describes the neutrino DIS data along with the charged-lepton data and, consequently, $\ell^{ \pm} \mathrm{Fe}$ and $v \mathrm{Fe}$ based on the $\mathrm{NuTeV}$ results, have different nuclear correction factors.

A compromise solution between $v A$ and $\ell^{ \pm} A$ data can be found only if the full correlated systematic errors of the $v A$ data are not used and the statistical and all systematic errors are combined in quadrature thereby neglecting the information contained in the correlation matrix. In other words the larger errors resulting from combining statistical and all systematic errors in quadrature reduces the discriminatory power of the fit such that the difference between $v A$ and $\ell^{ \pm} A$ data are no longer evident. This conclusion underscores the fundamental difference [129] of the nCTEQ analysis with other contemporary analyses.

On the other hand, a difference between $v A$ and $\ell^{ \pm} A$ is not completely unexpected, particularly in the shadowing region, and has previously been discussed in the literature $[136,137]$. The charged-lepton processes occur (dominantly) via $\gamma$-exchange, while the neutrino-nucleon processes occur via $W^{ \pm}$-exchange. The different nuclear corrections could simply be a consequence of the differing propagation of the hadronic fluctuations of the intermediate bosons (photon, $W$ ) through dense nuclear matter. Furthermore, since the 
structure functions in neutrino DIS and charged lepton DIS are distinct observables with different parton model expressions, it is clear that the nuclear correction factors will not be exactly the same. What is, however, unexpected is the degree to which the $R$ factors differ between the structure functions $F_{2}^{\nu \mathrm{Fe}}$ and $F_{2}^{\ell \mathrm{Fe}}$. In particular the lack of evidence for shadowing in neutrino scattering at low $Q^{2}$ down to $x \sim 0.02$ is quite surprising.

Should subsequent experimental results confirm the rather substantial difference between charged-lepton and neutrino scattering in the shadowing region at low- $Q^{2}$ it is interesting to speculate on the possible cause of the difference. A recent study of EMC, BCDMS and NMC data by a Hampton University, Jefferson Laboratory collaboration [138] suggests that anti-shadowing in charged-lepton nucleus scattering may be dominated by the longitudinal structure function $F_{L}$. As a by-product of this study, their figures hint that shadowing in the data of EMC, BCDMS, and NMC $\mu A$ scattering was being led by the transverse cross section with the longitudinal component crossing over into the shadowing region at lower $x$ compared to the transverse.

As summarized earlier, in the low- $Q^{2}$ region, the neutrino cross section is dominated by the longitudinal structure function $F_{L}$ via axial-current interactions since $F_{T}$ vanishes as $Q^{2}$ as $Q^{2} \rightarrow 0$ similar to the behavior of charged lepton scattering. If the results of the $\mathrm{NuTeV}$ analysis are verified, one contribution to the different behavior of shadowing at low$Q^{2}$ demonstrated by $v A$ and $\ell A$, in addition to the different hadronic fluctuations in the two interactions, could be due to the different mix of longitudinal and transverse contributions to the cross section of the two processes in this kinematic region.

Another hypothesis of what is causing the difference between neutrino and chargedlepton shadowing results comes from Guzey et al. [139] who speculates that at low $x$, low- $Q^{2}$ the value of $y$ is close to unity and the neutrino interactions primarily probe the down and strange quarks. This is very different than the situation with charged-lepton scattering where the contribution from down and strange quarks are suppressed by a factor of $1 / 4$ compared to the up and charm. Therefore, the discrepancy between the observed nuclear shadowing in lepton-nucleus total cross section at small $x$ and shadowing in total neutrino-nucleus cross section could be caused by the absence of nuclear shadowing in the nuclear strange quark distributions as extracted from the neutrino-nucleus data or even the poor knowledge of the strange-quark distribution in the free-nucleon that affects the neutrino-nucleus ratio more than the charged-lepton ratio.

\section{Acknowledgments}

This research was supported by DGI and FEDER funds, under Contracts FIS201128853-C02-02 and the Spanish Consolider-Ingenio 2010 Programme CPAN (CSD200700042), by Generalitat Valenciana under Contract PROMETEO/2009/0090, and by the EU HadronPhysics2 project, Grant agreement no. 227431. J. T. Sobczyk (on leave from Wrocław University and partially supported by Grants NN202 368439 and DWM/57/T2 K/2007)

thanks T. Golan for making Figure 1. Fermilab is operated by Fermi Research Alliance, LLC under Contract no. De-AC02-07CH11359 with the United States Department of Energy.

\section{References}

[1] H. Gallagher, G. Garvey, and G. P. Zeller, "Neutrino-nucleus interactions," Annual Review of Nuclear and Particle Science, vol. 61, pp. 355-378, 2011.

[2] Q. Wu, S. R. Mishra, A. Godley et al., "A precise measurement of the muonneutrino-nucleon inclusive charged current cross section off an isoscalar target in the energy range $2.5<E_{v}<40$ by NOMAD," Physics Letters B, vol. 660, no. 1-2, pp. 19-25, 2008. 
[3] P. Adamson, C. Andreopoulos, K. E. Arms et al., "Neutrino and antineutrino inclusive chargedcurrent cross section measurements with the MINOS near detector," Physical Review D, vol. 81, no. 7, Article ID 072002, 16 pages, 2010.

[4] Y. Nakajima, J. L. Alcaraz-Aunion, S. J. Brice et al., "Measurement of inclusive charged current interactions on carbon in a few-GeV neutrino beam," Physical Review D, vol. 83, no. 1, Article ID 012005, 21 pages, 2011.

[5] M. Ravonel Salzgeber, "Measurement of the flux averaged Inclusive Charged Current cross section," in A Poster Presented at International Conference on Neutrino Physics and Astrophysics (NEUTRINO '12), Kyoto, Japan, June 2012.

[6] B. Bhattacharya, R. J. Hill, and G. Paz, "Model independent determination of the axial mass parameter in quasielastic neutrino-nucleon scattering," Physical Review D, vol. 84, no. 7, Article ID 073006, 10 pages, 2011.

[7] A. Bodek, S. Avvakumov, R. Bradford, and H. S. Budd, "Extraction of the axial nucleon form-factor from neutrino experiments on deuterium," The European Physical Journal C, vol. 63, p. 355, 2009.

[8] V. Bernard, L. Elouadrhiri, and U. G. Meissner, "Axial structure of the nucleon: topical review," Journal of Physics G, vol. 28, no. 1, p. R1, 2002.

[9] R. Gran, E. J. Jeon et al., "Measurement of the quasielastic axial vector mass in neutrino interactions on oxygen," Physical Review D, vol. 74, no. 6, Article ID 052002, 15 pages, 2006.

[10] X. Espinal and F. Sanchez, "Measurement of the axial vector mass in neutrino-carbon interactions at K2K," AIP Conference Proceedings, vol. 967, no. 1, p. 117, 2007.

[11] M. Dorman, "Preliminary results for CCQE scattering with the MINOS near detector," in AIP Conference Proceedings, vol. 1189, pp. 133-138, 2009.

[12] A. A. Aguilar-Arevalo, C. E. Anderson, A. O. Bazarko et al., "First measurement of the muon neutrino charged current quasielastic double differential cross section," Physical Review D, vol. 81, no. 9, Article ID 092005, 22 pages, 2010.

[13] V. Lyubushkin, B. Popov, J. J. Kim et al., "Study of quasi-elastic muon neutrino and antineutrino scattering in the NOMAD experiment," The European Physical Journal C, vol. 63, no. 3, pp. 355-381, 2009.

[14] N. Mayer and N. Graf, "Improvements to MINOS CCQE analysis," in AIP Conference Proceedings, vol. 1405, pp. 41-46, 2011.

[15] Y. Nakajima, "Measurement of CC and CCQE interactions at SciBooNE," in AIP Conference Proceedings, vol. 1405, pp. 47-52, 2011.

[16] L. Alcaraz Aunion, "Measurement of the absolute $v_{\mu}$-CCQE cross section at the Sci- BooNE experiment," PhD Thesis supervised by F. Sánchez, Barcelona, July 2010, http://lss.fnal.gov/archive/ thesis / fermilab-thesis-2010-45.pdf.

[17] J. Grange, "New results from MiniBooNE charged-current quasi-elastic anti-neutrino data," in AIP Conference Proceedings, vol. 1405, p. 83, 2011.

[18] J. G. Morfin, "Inclusive neutrino cross section measurements at MINERvA," in A Poster Presented at International Conference International Conference on Neutrino Physics and Astrophysics (NEUTRINO '12), AIP Conference Proceedings, pp. 243-248, Kyoto, Japan, June 2012.

[19] D. Casper, "The Nuance neutrino physics simulation, and the future," Nuclear Physics B (Proceedings Supplement), vol. 112, no. 1-3, pp. 161-170, 2002.

[20] E. Oset and L. L. Salcedo, " $\Delta$ self-energy in nuclear matter," Nuclear Physics A, vol. 468, no. 3-4, pp. 631-652, 1987.

[21] J. A. Nowak, "Four momentum transfer discrepancy in the charged current $\pi^{+}$production in the MiniBooNE: data vs. theory," in Proceedings of the 6th International Workshop on Neutrino-Nuceus Interactions in the Few-Gev Region (NUINT '09), vol. 1189 of AIP Conference Proceedings, pp. 243-248, 2009.

[22] S. Boyd, S. Dytman, E. Hernandez, J. Sobczyk, and R. Tacik, "Comparison of models of neutrinonucleus interactions," in AIP Conference Proceedings, vol. 1189, pp. 60-73, 2009.

[23] R. A. Smith and E. J. Moniz, "Neutrino reactions on nuclear targets," Nuclear Physics B, vol. 43, pp. 605-622, 1972.

[24] R. A. Smith and E. J. Moniz, "Erratum: neutrino reactions on nuclear targets," Nuclear Physics B, vol. 101, p. $547,19$.

[25] O. Benhar, A. Fabrocini, S. Fantoni, and I. Sick, "Spectral function of finite nuclei and scattering of GeV electrons," Nuclear Physics A, vol. 579, no. 3-4, pp. 493-517, 1994.

[26] O. Benhar and D. Meloni, "Total neutrino and antineutrino nuclear cross sections around $1 \mathrm{GeV}$," Nuclear Physics A, vol. 789, no. 1-4, pp. 379-402, 2007. 
[27] A. M. Ankowski and J. T. Sobczyk, "Construction of spectral functions for medium-mass nuclei," Physical Review C, vol. 77, no. 4, Article ID 044311, 14 pages, 2008.

[28] T. Leitner, O. Buss, L. Alvarez-Ruso, and U. Mosel, "Electron- and neutrino-nucleus scattering from the quasielastic to the resonance region," Physical Review C, vol. 79, no. 3, Article ID 034601, 26 pages, 2009.

[29] J. Nieves, J. E. Amaro, and M. Valverde, "Inclusive quasi-elastic neutrino reactions," Physical Review C, vol. 70, Article ID 055503, 2004.

[30] J. Nieves, J. E. Amaro, and M. Valverde, "Erratum: inclusive quasi-elastic neutrino reactions," Physical Review C, vol. 72, Article ID 019902, 2005.

[31] J. Nieves, M. Valverde, and M. J. Vicente Vacas, “Inclusive nucleon emission induced by quasielastic neutrino-nucleus interactions," Physical Review C, vol. 73, no. 2, Article ID 025504, 18 pages, 2006.

[32] M. Martini, M. Ericson, G. Chanfray, and J. Marteau, "A unified approach for nucleon knock-out, coherent and incoherent pion production in neutrino interactions with nuclei," Physical Review C, vol. 80, no. 6, Article ID 065501, 16 pages, 2009.

[33] M. Martini, M. Ericson, G. Chanfray, and J. Marteau, "Neutrino and antineutrino quasielastic interactions with nuclei," Physical Review C, vol. 81, no. 4, Article ID 045502, 5 pages, 2010.

[34] M. Sajjad Athar, S. Chauhan, and S. K. Singh, "Theoretical study of lepton events in the atmospheric neutrino experiments at SuperK," The European Physical Journal A, vol. 43, no. 2, pp. 209-227, 2010.

[35] D. H. Wilkinson, "Renormalization of the axial-vector coupling constant in nuclear $\beta$-decay (II)," Nuclear Physics A, vol. 209, no. 3, pp. 470-484, 1973.

[36] D. H. Wilkinson, "Renormalization of the axial-vector coupling constant in nuclear $\beta$-decay (III)," Nuclear Physics A, vol. 225, no. 3, pp. 365-381, 1974.

[37] J. Nieves, I. Ruiz Simo, and M. J. Vicente Vacas, "The nucleon axial mass and the MiniBooNE Quasielastic Neutrino-Nucleus Scattering problem," Physics Letters B, vol. 707, p. 72, 2012.

[38] M. Martini, M. Ericson, and G. Chanfray, "Neutrino quasielastic interaction and nuclear dynamics," Physical Review C, vol. 84, Article ID 055502, 2011.

[39] A. A. Aguilar-Arevalo et al., "The Neutrino Flux prediction at MiniBooNE," Physical Review D, vol. 79, no. 7, Article ID 072002, 38 pages, 2009.

[40] N. Jachowicz, C. Praet, and J. Ryckebusch, "Modeling neutrino-nucleus interactions in the few-GeV regime," Acta Physica Polonica B, vol. 40, no. 9, pp. 2559-2564, 2009.

[41] N. Jachowicz, P. Vancraeyveld, P. Lava, C. Praet, and J. Ryckebusch, "Strangeness content of the nucleon in quasielastic neutrino-nucleus reactionsof the nucleon in quasielastic neutrino-nucleus reactions," Physical Review C, vol. 76, no. 5, Article ID 055501, 11 pages, 2007.

[42] C. Maieron, M. C. Martinez, J. A. Caballero, and J. M. Udias, "Nuclear model effects in charged current neutrino nucleus quasielastic scattering," Physical Review C, vol. 68, Article ID 048501, 2003.

[43] J. E. Amaro, M. B. Barbaro, J. A. Caballero, T. W. Donnelly, and J. M. Udias, "Relativistic analyses of quasielastic neutrino cross sections at MiniBooNE kinematics," Physical Review D, vol. 84, no. 3, Article ID 033004, 8 pages, 2011.

[44] B. Serot and J. Walecka, "The relativistic many-body problem," Advances in Nuclear Physics, vol. 16, p. 1, 1986.

[45] A. Meucci, C. Giusti, and F. D. Pacati, "Relativistic descriptions of final-state interactions in neutralcurrent neutrino-nucleus scattering at MiniBooNE kinematics," Physical Review D, vol. 84, Article ID 113003, 2011.

[46] T. W. Donnelly and I. Sick, "Superscaling of inclusive electron scattering from nuclei," Physical Review Letters, vol. 82, no. 16, pp. 3212-3215, 1999.

[47] T. W. Donnelly and I. Sick, "Superscaling in inclusive electron-nucleus scattering," Physical Review C, vol. 60, Article ID 065502, 1999.

[48] J. E. Amaro, M. B. Barbaro, J. A. Caballero, T. W. Donnelly, A. Molinari, and I. Sick, “Using electron scattering superscaling to predict charge-changing neutrino cross sections in nuclei," Physical Review C, vol. 71, no. 1, Article ID 015501, 17 pages, 2005.

[49] J. E. Amaro, M. B. Barbaro, J. A. Caballero, and T. W. Donnelly, "Superscaling and neutral current quasielastic neutrino-nucleus scattering beyond the relativistic fermi gas model," Physical Review C, vol. 73, no. 6, Article ID 035503, 8 pages, 2006.

[50] L. Alvarez-Ruso, "Neutrino interactions: challenges in the current theoretical picture," Nuclear Physics B (Proceedings Supplement), vol. 229-232, pp. 167-173, 2012.

[51] O. Benhar and D. Meloni, "Impact of nuclear effects on the determination of the nucleon axial mass," Physical Review D, vol. 80, no. 7, Article ID 073003, 6 pages, 2009. 
[52] O. Benhar, P. Coletti, and D. Meloni, "Electroweak nuclear response in quasi-elastic regime," Physical Review Letters, vol. 105, no. 13, Article ID 132301, 4 pages, 2010.

[53] C. Juszczak, J. T. Sobczyk, and J. Żmuda, "Extraction of the axial mass parameter from MiniBooNE neutrino quasielastic double differential cross-section data," Physical Review C, vol. 82, no. 4, Article ID 045502, 6 pages, 2010.

[54] A. V. Butkevich, "Analysis of flux-integrated cross sections for quasi-elastic neutrino charged-current scattering off ${ }^{12} \mathrm{C}$ at MiniBooNE energies," Physical Review C, vol. 82, Article ID 055501, 2010.

[55] J. Marteau, "Effects of the nuclear correlations on the neutrino-oxygen interactions," The European Physical Journal A, vol. 5, no. 2, pp. 183-190, 1999.

[56] J. Nieves, I. Ruiz Simo, and M. J. Vicente Vacas, "Inclusive charged—current neutrino-nucleus reactions," Physical Review C, vol. 83, Article ID 045501, 2011.

[57] W. M. Alberico, M. Ericson, and A. Molinari, "The role of two particle-two hole excitations in the spin-isospin nuclear response," Annals of Physics, vol. 154, no. 2, pp. 356-395, 1984.

[58] J. E. Amaro, M. B. Barbaro, J. A. Caballero, T. W. Donnelly, and C. F. Williamson, "Meson-exchange currents and quasielastic neutrino cross sections in the Super Scaling Approximation model," Physics Letters B, vol. 696, p. 151, 2011.

[59] J. E. Amaro, M. B. Barbaro, J. A. Caballero, and T. W. Donnelly, "Meson-exchange currents and quasielastic antineutrino cross sections in the SuperScaling Approximation," Physical Review Letters, vol. 108, Article ID 152501, 2012.

[60] A. Bodek, H. S. Budd, and M. E. Christie, "Neutrino quasielastic scattering on nuclear targets parametrizing transverse enhancement (meson exchange currents)," The European Physical Journal C, vol. 71, no. 9, p. 1726, 2011.

[61] O. Lalakulich, K. Gallmeister, and U. Mosel, "Many-body interactions of neutrinos with nucleiobservables," Physical Review C, vol. 86, Article ID 014614, 2012.

[62] J. T. Sobczyk, "Multinucleon ejection model for Meson Exchange Current neutrino interactions," Physical Review C, vol. 86, Article ID 015504, 2012.

[63] P. de Perio, "NEUT pion FSI," in Proceedings of the 7th International Workshop on Neutrino-Nucleus Interactions in the Few GeV Region (NuInt '11), vol. 1405 of AIP Conference Proceedings, p. 223, Dehradun, India, March 2011.

[64] T. Golan, C. Juszczak, and J. T. Sobczyk, "Final state interactions effects in neutrino-nucleus interactions," Physical Review C, vol. 86, Article ID 015505, 2012.

[65] D. Meloni and M. Martini, "Revisiting the T2K data using different models for the neutrino-nucleus cross sections," Physics Letters B, vol. 716, p. 186, 2012.

[66] M. Martini, M. Ericson, and G. Chanfray, "Neutrino energy reconstruction problems and neutrino oscillations," Physical Review D, vol. 85, Article ID 09312, 2012.

[67] J. Nieves, F. Sanchez, I. Ruiz Simo, and M. J. Vicente Vacas, “Neutrino energy reconstruction and the shape of the CCQE-like total cross section," Physical Review D, vol. 85, Article ID 113008, 2012.

[68] U. Mosel and O. Lalakulich, "Neutrino-long-baseline experiments and nuclear physics," in Proceedings of 50th International Winter Meeting on Nuclear Physics (PoS BORMIO '12), Bormio, Italy, January 2012, 034.

[69] A. A. Aguilar-Arevalo, C. E. Anderson, A. O. Bazarko et al., "Measurement of the neutrino neutralcurrent elastic Differential cross section," Physical Review D, vol. 82, no. 9, Article ID 092005, 16 pages, 2010.

[70] A. V. Butkevich and D. Perevalov, "Neutrino neutral-current elastic scattering on ${ }^{12}$ C," Physical Review C, vol. 84, no. 1, Article ID 015501, 9 pages, 2011.

[71] O. Benhar and G. Veneziano, "Nuclear effects in neutral current quasi-elastic neutrino interactions," Physics Letters B, vol. 702, p. 326, 2011.

[72] A. M. Ankowski, "Consistent analysis of neutral- and charged-current neutrino scattering off carbon," Physical Review C, vol. 86, no. 2, Article ID 024616, 13 pages, 2012.

[73] A. A. Aguilar-Arevalo, C. E. Anderson, A. O. Bazarko et al., "First observation of coherent $\pi^{0}$ production in neutrino-nucleus interactions with $E_{v}<2 \mathrm{GeV}$," Physics Letters B, vol. 664, no. 1-2, pp. 41-46, 2008.

[74] S. Nakayama, S. Nakayama, C. Mauger et al., "Measurement of the single pi0 production in neutral current neutrino interactions with water by a $1.3 \mathrm{GeV}$ wide band muon neutrino beam," Physics Letters B, vol. 619, no. 3-4, pp. 255-262, 2005.

[75] A. A. Aguilar-Arevalo, C. E. Anderson, A. O. Bazarko et al., "Measurement of $v_{\mu}$ and $\bar{v}_{\mu}$ induced neutral current single $\pi^{0}$ production cross sections on mineral oil at $E_{v} \sim O(1 \mathrm{GeV})$, , Physical Review D, vol. 81, Article ID 013005, 2010. 
[76] Y. Kurimoto, J. L. Alcaraz-Aunion, S. J. Brice et al., "Measurement of inclusive neutral current neutral pion production on carbon in a Few-GeV neutrino beam," Physical Review D, vol. 81, Article ID 033004, 2009.

[77] A. A. Aguilar-Arevalo, C. E. Anderson, A. O. Bazarko et al., "Measurement of neutrino-induced charged-current charged pion production cross sections on mineral oil at $E_{v} 1 \mathrm{GeV}$, ," Physical Review D, vol. 83, Article ID 052007, 2011.

[78] O. Lalakulich, K. Gallmeister, T. Leitner, and U. Mosel, "Pion production in the MiniBooNE," in Proceedings of the 7th International Workshop on Neutrino-Nucleus Interactions in the Few GeV Region (NuInt '11), vol. 1405 of AIP Conference Proceedings, Dehradun, India, March 2011.

[79] A. A. Aguilar-Arevalo, C. E. Anderson, A. O. Bazarko et al., "Measurement of $v_{\mu}$-induced chargedcurrent neutral pion production cross sections on mineral oil at $E_{v} \in 0.5-2.0 \mathrm{GeV}$," Physical Review D, vol. 83, no. 5, Article ID 052009, 2011.

[80] A. A. Aguilar-Arevalo et al., "Measurement of the $v_{\mu} C C \pi^{+} / \mathrm{QE}$ cross section ratio on mineral oil in a $0.8 \mathrm{GeV}$ neutrino beam," Physical Review Letters, vol. 103, Article ID 081801, 2009.

[81] A. Rodriguez, L. Whitehead, J. L. Alcaraz Aunion et al., "Measurement of single charged pion production in the charged-current interactions of neutrinos in a $1.3 \mathrm{GeV}$ wide band beam," Physical Review D, vol. 78, no. 3, Article ID 032003, 2008.

[82] T. Leitner, O. Buss, L. Alvarez-Ruso, and U. Mosel, "Electron- and neutrino-nucleus scattering from the quasielastic to the resonance region," Physical Review C, vol. 79, no. 3, Article ID 034601, 26 pages, 2009.

[83] T. Leitner, O. Buss, U. Mosel, and L. Alvarez-Ruso, "Neutrino-induced pion production at energies relevant for the MiniBooNE and K2K experiments," Physical Review C, vol. 79, no. 3, Article ID 038501, 2009.

[84] M. Sajjad Athar, S. Chauhan, and S. K. Singh, "CC1 $\pi^{+}$to CCQE cross sections ratio at accelarator energies," Journal of Physics G, vol. 37, no. 1, Article ID 015005, 2010.

[85] M. J. Vicente Vacas, M. Kh. Khankhasaev, and S. G. Mashnik, "Inclusive pion double-chargeexchange above $.5 \mathrm{GeV}$," http://arxiv.org/abs/nucl-th/9412023.

[86] S. K. Singh, M. Sajjad Athar, and S. Ahmad, "Nuclear effects in neutrino induced coherent pion production at K2K and MiniBooNE," Physical Review Letters, vol. 96, no. 24, Article ID 241801, 4 pages, 2006.

[87] K. M. Graczyk, D. Kiełczewska, P. Przewłocki, and J. T. Sobczyk, " $C_{5}^{A}$ axial form factor from bubble chamber experiments," Physical Review D, vol. 80, no. 9, Article ID 093001, 14 pages, 2009.

[88] E. Hernandez, J. Nieves, and M. Valverde, "Weak pion production off the nucleon," Physical Review D, vol. 76, no. 3, Article ID 033005, 22 pages, 2007.

[89] O. Lalakulich and E. A. Paschos, "Resonance production by neutrinos: $J=3 / 2$ resonances," Physical Review D, vol. 71, no. 7, Article ID 074003, 10 pages, 2005.

[90] O. Lalakulich, T. Leitner, O. Buss, and U. Mosel, "One pion production in neutrino reactions: including non-resonant background," Physical Review D, vol. 82, no. 9, Article ID 093001, 18 pages, 2010.

[91] L. Alvarez-Ruso, S. K. Singh, and M. J. Vicente Vacas, "Neutrino $d \rightarrow \mu^{-} \Delta^{++} n$ reaction and axial vector N $\Delta$ coupling," Physical Review C, vol. 59, Article ID 3386, 1999.

[92] M. Maltoni and T. Schwetz, "Testing the statistical compatibility of independent data sets," Physical Review D, vol. 68, no. 3, Article ID 033020, 9 pages, 2003.

[93] E. Hernandez, J. Nieves, M. Valverde, and M. J. Vicente Vacas, " $N-\Delta(1232)$ axial form factors from weak pion production," Physical Review D, vol. 81, no. 8, Article ID 085046, 5 pages, 2010.

[94] T. Sato, D. Uno, and T. S. H. Lee, "Dynamical model of weak pion production reactions," Physical Review C, vol. 67, no. 6, Article ID 065201, 13 pages, 2003.

[95] C. Barbero, G. Lopez Castro, and A. Mariano, "Single pion production in CC $v_{\mu} N$ scattering within a consistent effective Born approximation," Physics Letters B, vol. 664, pp. 70-77, 2008.

[96] Y. Kurimoto, J. L. Alcaraz Aunion, S. J. Brice et al., "Improved measurement of neutral current coherent $\pi^{0}$ production on carbon in a Few-GeV neutrino beam," Physical Review D, vol. 81, Article ID 111102, 2010.

[97] M. Hasegawa, E. Aliu, S. Andringa et al., "Search for coherent charged pion production in neutrinocarbon interactions," Physical Review Letters, vol. 95, no. 25, Article ID 252301, 5 pages, 2005.

[98] K. Hiraide, J. L. Alcaraz Aunion, S. J. Brice et al., "Search for charged current coherent pion production on carbon in a Few-GeV neutrino beam," Physical Review D, vol. 78, no. 11, Article ID 112004, 19 pages, 2008. 
[99] D. Rein and L. M. Sehgal, "Coherent pi0 production in neutrino reactions," Nuclear Physics B, vol. 223, article 29, 1983.

[100] Ch. Berger and L. M. Sehgal, "Lepton mass effects in single pion production by neutrinos," Physical Review D, vol. 76, no. 11, Article ID 113004, 8 pages, 2007.

[101] Ch. Berger and L. M. Sehgal, "Partially conserved axial vector current and coherent pion production by low energy neutrinos," Physical Review D, vol. 79, no. 5, Article ID 053003, 6 pages, 2009.

[102] E. A. Paschos and D. Schalla, "Coherent pion production by neutrinos," Physical Review D, vol. 80, Article ID 033005, 2009.

[103] E. Hernandez, J. Nieves, and M. J. Vicente-Vacas, "Neutrino induced coherent pion production off nuclei and the partial conservation of the axial current," Physical Review D, vol. 80, no. 1, Article ID 013003, 15 pages, 2009.

[104] S. X. Nakamura, T. Sato, T.-S. H. Lee, B. Szczerbinska, and K. Kubodera, "Dynamical model of coherent pion production in neutrino-nucleus scattering," Physical Review C, vol. 81, no. 3, Article ID 035502, 22 pages, 2010.

[105] J. E. Amaro, E. Hernandez, J. Nieves, and M. Valverde, "Theoretical study of neutrino-induced coherent pion production off nuclei at T2K and MiniBooNE energies," Physical Review D, vol. 79, Article ID 013002, 18 pages, 2009.

[106] E. Hernandez, J. Nieves, and M. Valverde, "Coherent pion production off nuclei at T2K and MiniBooNE energies revisited," Physical Review D, vol. 82, no. 7, Article ID 077303, 4 pages, 2010.

[107] L. Alvarez-Ruso, L. S. Geng, S. Hirenzaki, and M. J. Vicente Vacas, "Charged current neutrino induced coherent pion production," Physical Review C, vol. 75, no. 5, Article ID 055501, 8 pages, 2007.

[108] L. Alvarez-Ruso, L. S. Geng, S. Hirenzaki, and M. J. Vicente Vacas, "Erratum: charged current neutrino induced coherent pion production," Physical Review C, vol. 80, Article ID 019906, 2009.

[109] D. Rein and L. M. Sehgal, "Neutrino excitation of baryon resonances and single pion production," Annals of Physics, vol. 133, pp. 79-153, 1981.

[110] K. M. Graczyk and J. T. Sobczyk, "Lepton mass effects in weak charged current single pion production," Physical Review D, vol. 77, no. 5, Article ID 053003, 7 pages, 2008.

[111] K. M. Graczyk and J. T. Sobczyk, "Form factors in the quark resonance model," Physical Review D, vol. 77, no. 5, Article ID 053001, 12 pages, 2008.

[112] K. M. Graczyk and J. T. Sobczyk, "Erratum: form factors in the quark resonance model," Physical Review D, vol. 79, Article ID 079903, 2009.

[113] O. Lalakulich, W. Melinitchouk, and E. A. Paschos, "Quark-hadron duality in neutrino scattering," Physical Review C, vol. 75, no. 1, Article ID 015202, 10 pages, 2007.

[114] K. M. Graczyk, C. Juszczak, and J. T. Sobczyk, "Quark-hadron duality in the Rein-Sehgal model," Nuclear Physics A, vol. 781, no. 1-2, pp. 227-246, 2007.

[115] O. Lalakulich, N. Jachowicz, Ch. Praet, and J. Ryckebusch, "Quark-hadron duality in lepton scattering off nuclei," Physical Review C, vol. 79, no. 1, Article ID 015206, 12 pages, 2009.

[116] A. Bodek and U.-K. Yang, "NUFACT09 update to the Bodek-Yang unified model for electronand neutrino- nucleon scattering cross sections," in Proceedings of the11th International Workshop on Neutrino Factories, Superbeams and Beta Beams-NuFact, vol. 1222 of AIP Conference Proceedings, pp. 233-237, March 2010.

[117] M. Gluck, E. Reya, and A. Vogt, “Dynamical parton distributions revisited," The European Physical Journal C, vol. 5, no. 3, pp. 461-470, 1998.

[118] R. Thorne and R. Roberts, "Practical procedure for evolving heavy flavor structure functions," Physics Letters B, vol. 421, p. 303, 1998.

[119] A. D. Martin, R. G. Roberts, W. J. Stirling, R. S. Thorne et al., "Estimating the effect of NNLO contributions on global parton analyses," The European Physical Journal C, vol. 18, no. 1, pp. 117-126, 2000.

[120] L. W. Whitlow, S. Rock, A. Bodek et al., "A precise extraction of $R=\sigma_{L} / \sigma_{T}$ from a global analysis of the SLAC deep inelastic e-p and e-d scattering cross sections," Physics Letters B, vol. 250, no. 1-2, pp. 193-198, 1990.

[121] M. Tzanov, D. Naples, S. Boyd et al., "Precise measurement of neutrino and anti-neutrino differential cross sections," Physical Review D, vol. 74, no. 1, Article ID 012008, 16 pages, 2006.

[122] G. Onengut, R. Van Dantzig, M. De Jong et al., "Measurement of nucleon structure functions in neutrino scattering," Physics Letters B, vol. 632, pp. 65-75, 2006.

[123] J. Altegoer, M. Anfrevilleb, C. Angelini et al., "The NOMAD experiment at the CERN SPS," Nuclear Instruments and Methods in Physics Research Section A, vol. 404, no. 1, pp. 96-128, 1998. 
[124] R. Petti, "Cross-section measurements in the NOMAD experiment," Nuclear Phycics B (Proceedings Supplement), vol. 159, pp. 56-62, 2006.

[125] A. Accardi, M. E. Christy, C. E. Keppel et al., "New parton distributions from large-x and low-Q2 data," Physical Review D, vol. 81, no. 3, Article ID 034016, 2010.

[126] J. Pumplin, D. R. Stump, J. Huston, H. L. Lai, P. M. Nadolsky, and W. K. Tung, "New generation of parton distributions with uncertainties from global QCD analysis," JHEP, vol. 0207, p. 012, 2002.

[127] W. J. Stirling, A. D. Martin, R. G. Roberts, and R. S. Thorne, "MRST parton distributions," in AIP Conference Proceedings, vol. 747, p. 16, 2005.

[128] M. Tzanov, "NuTeV structure function measurement," in Proceedings of the 13th International Workshop on Deep Inelastic Scattering, vol. 792 of AIP Conference Proceedings, pp. 241-244, 2005.

[129] K. Kovarik, I. Schienbein, F. I. Olness et al., "Nuclear corrections in neutrino-nucleus DIS and their compatibility with global nPDF analyses," Physical Review Letters, vol. 106, no. 12, Article ID 122301, 4 pages, 2011.

[130] I. Schienbein, J. Y. Yu, C. Keppel, J. G. Morfin, F. Olness, and J. F. Owens, “Nuclear PDFs from neutrino deep inelastic scattering," Physical Review D, vol. 77, Article ID 054013, 2008.

[131] J. F. Owens, J. Huston, J. Pumplin et al., "Nuclear corrections and parton distribution functions: lessons learned from global fitting," in AIP Conference Proceedings, vol. 967, pp. 259-263, 2007.

[132] B. Z. Kopeliovich, J. G. Morfin, and I. Schmidt, "Nuclear shadowing in electro-weak interactions," Progress in Particle and Nuclear Physics, vol. 68, pp. 314-372, 2013.

[133] http://www.praktika.physik.uni-bonn.de/conferences/dis-2012?set_language=en.

[134] J. Botts, J. G. Morfin, J. F. Owens, J. Qiud, W.-K. Tunga, H. Weerts et al., "CTEQ parton distributions and flavor dependence of sea quarks," Physics Letters B, vol. 304, no. 1-2, pp. 159-166, 1993.

[135] H. L. Lai, J. Botts, J. Huston et al., "Global QCD analysis and the CTEQ parton distributions," Physical Review D, vol. 51, no. 9, pp. 4763-4782, 1995.

[136] S. J. Brodsky, I. Schmidt, and J. J. Yang, "Nuclear antishadowing in neutrino deep inelastic scattering," Physical Review D, vol. 70, Article ID 116003, 2004.

[137] J.-W. Qiu and I. Vitev, "Nuclear shadowing in neutrino nucleus deeply inelastic scattering," Physics Letters B, vol. 587, pp. 52-61, 2004.

[138] V. Guzey, L. Zhu, C. Keppel et al., "Impact of nuclear dependence of $R=\sigma_{L} / \sigma_{T}$ on antishadowing in nuclear structure functions," Physical Review C, vol. 86, no. 4, Article ID 045201, 9 pages, 2012.

[139] V. Guzey et al., "Nuclear shadowing in charged-lepton-nucleus and neutrino-nucleus scattering," Work in Progress. 

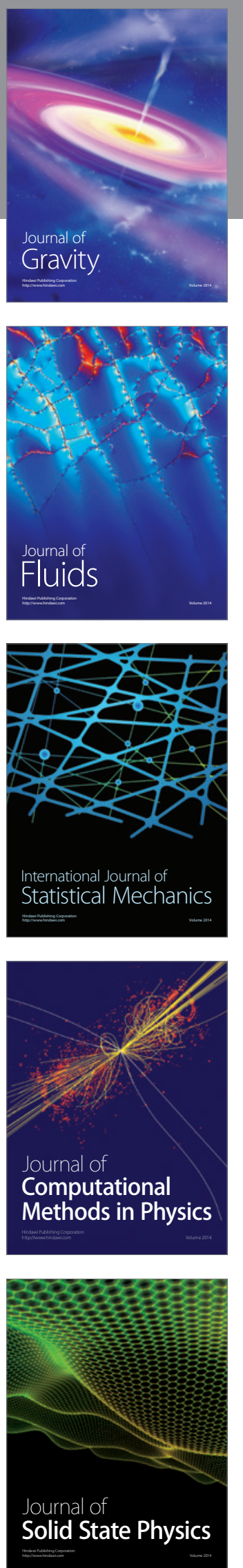

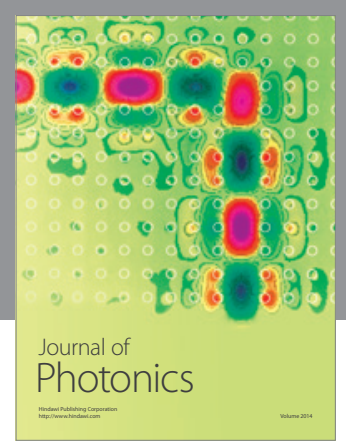

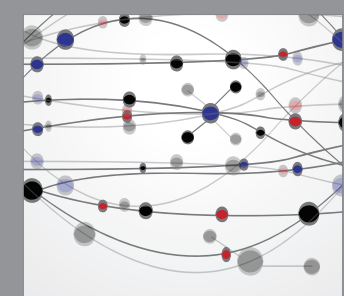

The Scientific World Journal
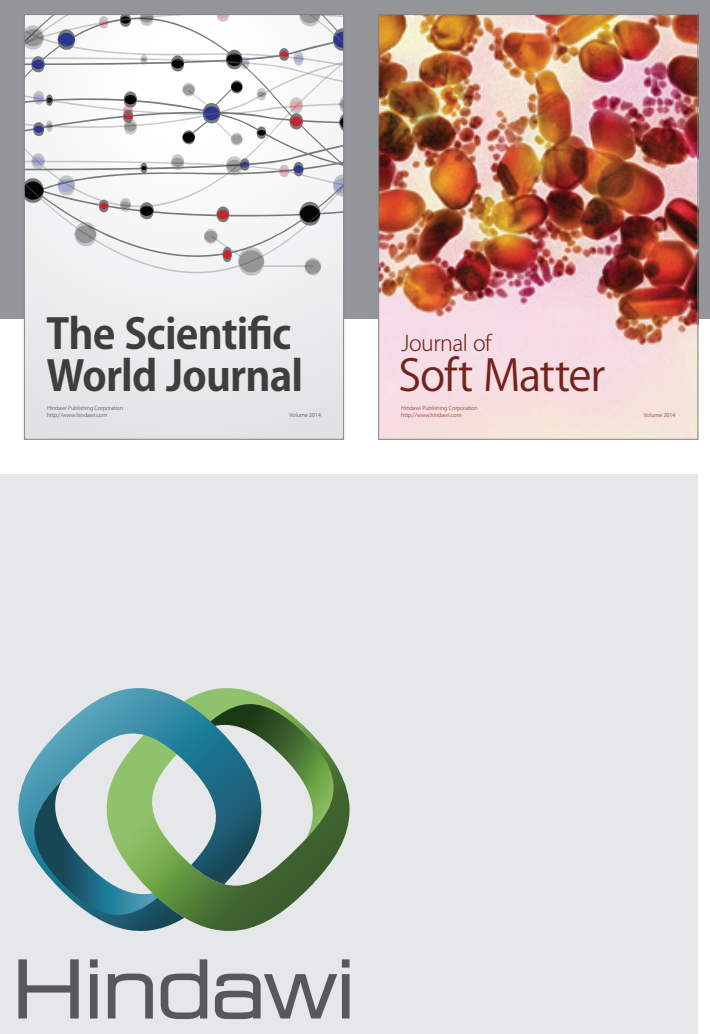

Submit your manuscripts at

http://www.hindawi.com
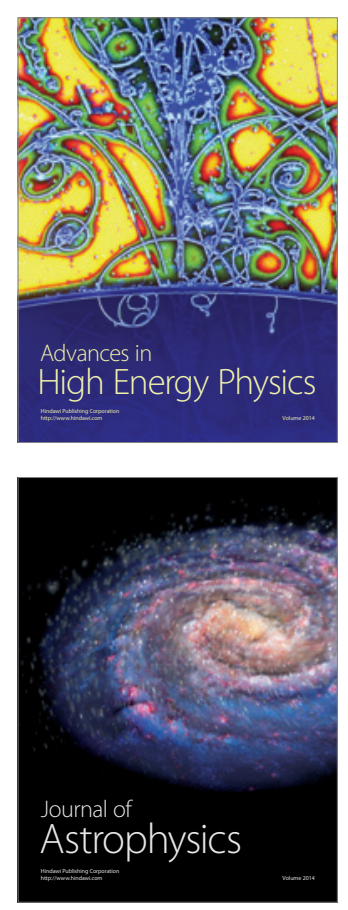
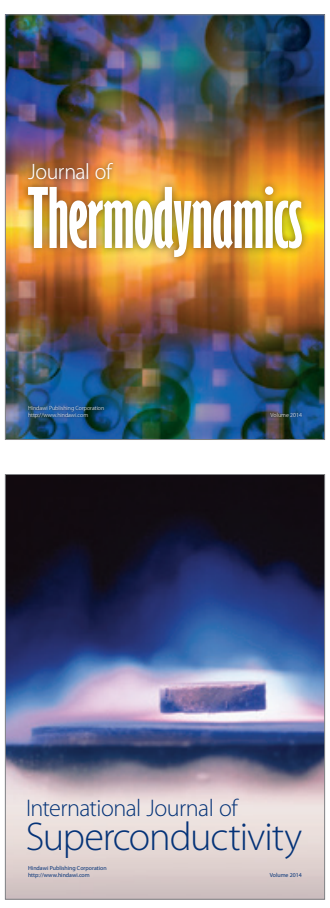
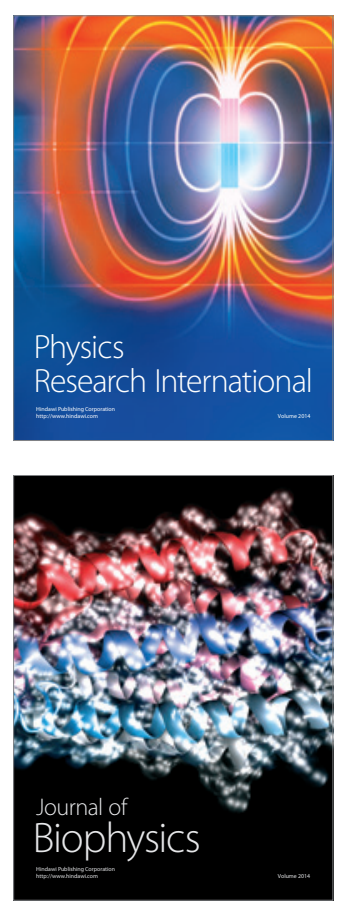
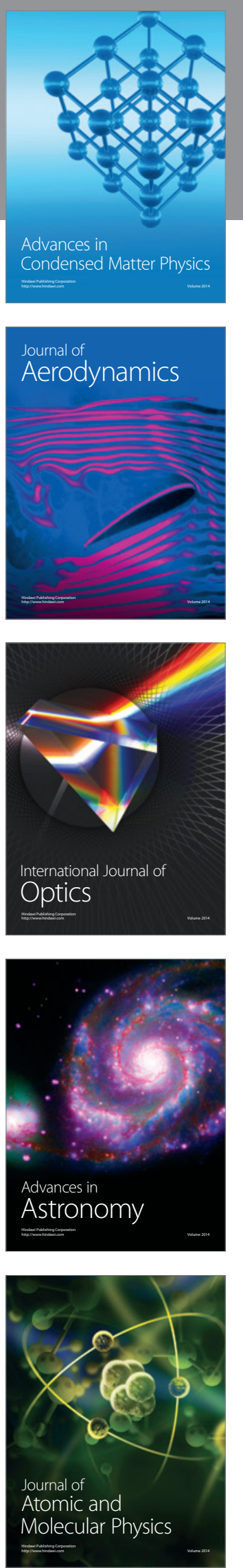